\title{
The use of mineral interfaces in sand-sized volcanic rock fragments to infer mechanical durability
}

\author{
Emilia Le Pera ${ }^{*}$ (i) and Consuele Morrone
}

\begin{abstract}
The use of mineral interfaces, in sand-sized rock fragments, to infer the influence exerted by mechanical durability on the generation of siliciclastic sediments, has been determined for plutoniclastic sand. Conversely, for volcaniclastic sand, it has received much less attention, and, to our knowledge, this is the first attempt to make use of the volcaniclastic interfacial modal mineralogy of epiclastic sandy fragments, to infer mechanical durability control at a modern beach environment. Volcaniclastic sand was collected along five beaches developed on five islands, of the southern Tyrrhenian Sea (Alicudi, Filicudi, Salina, Panarea and Stromboli) from the Aeolian Archipelago, and one sample was collected near the Stromboli Island volcanic crater. Each sample was sieved and thin sectioned for petrographic analysis. The modal mineralogy of the very coarse, coarse and medium sand fractions was determined by point-counting of the interfacial boundaries discriminating 36 types of interfaces categories, both no-isomineralic and/or no iso-structural (e.g., phenocrystal/glassy groundmass or phenocrystal/ microlitic groundmass boundaries) and iso-mineralic interfaces, inside volcanic lithic grains with lathwork and porphyric textures. A total of 47,386 interfacial boundaries have been counted and, the most representative series of interfaces, from the highest to the lowest preservation, can be grouped as: a) ultrastable interfaces, categorized as $\mathrm{Pl}$ (Plagioclase)/Glgr (Glassy groundmass) $>>P x$ (Pyroxene)/Glgr $>>\mathrm{Ol}$ (Olivine)/Glgr $>>\mathrm{Op}($ Opaque)/Glgr $>>\mathrm{Hbl}$ (Hornblende)/Glgr $>>B t$ (Biotite)/Glgr > >ldd (Iddingsite)/Glgr >>Rt (Rutile) / Glgr; b) stable interfaces, categorized as Pl/Migr (Microlitic groundmass) >>Op/Migr >>Px/Migr >>OI/Migr; c) moderately stable interfaces, categorized as $\mathrm{Op} / \mathrm{Px}>>\mathrm{Op} / \mathrm{Hbl}>>\mathrm{PX} / \mathrm{P}>>\mathrm{O} / \mathrm{Pl}>>\mathrm{Bt} / \mathrm{Op}$; and d) unstable interfaces, categorized as $\mathrm{PI} / \mathrm{PI}>>\mathrm{Px} / \mathrm{Px}>>\mathrm{O} / / \mathrm{Ol}>>$ $\mathrm{Op} / \mathrm{Op}>>\mathrm{Hbl/Hbl}>>\mathrm{Bt} / \mathrm{Bt}$. Grains, eroded from the volcanic bedrock, if affected solely by abrasion, developed a rounded and smoothed form, with prevailing no-isostructural interfaces such as Plagioclase/Glassy groundmass, Pyroxene/Glassy groundmass and Olivine/Glassy groundmass interfaces. Grains that during transport suffered fracturing and percussion have a sharp and angular form: these combined transport mechanisms produce mainly volcanic sandy grains with iso-structural interfaces, such as $\mathrm{Pl} / \mathrm{PI}, \mathrm{Px} / \mathrm{Px}, \mathrm{Hbl} / \mathrm{Hbl}$, and, to a lesser extent, Bt/Op and $\mathrm{Bt} / \mathrm{Glgr}$ interfaces.
\end{abstract}

Keywords: Volcaniclastic sand, Mineral interfaces, Volcanic beach environment, Mechanical durability

\footnotetext{
* Correspondence: emilia.lepera@unical.it

Dipartimento di Biologia, Ecologia e Scienze della Terra (DiBEST), Università

della Calabria, via P. Bucci, cubo 15b, 87036 Rende, CS, Italy
}

\section{Springer Open}

(c) The Author(s). 2020 Open Access This article is licensed under a Creative Commons Attribution 4.0 International License, which permits use, sharing, adaptation, distribution and reproduction in any medium or format, as long as you give appropriate credit to the original author(s) and the source, provide a link to the Creative Commons licence, and indicate if changes were made. The images or other third party material in this article are included in the article's Creative Commons licence, unless indicated otherwise in a credit line to the material. If material is not included in the article's Creative Commons licence and your intended use is not permitted by statutory regulation or exceeds the permitted use, you will need to obtain permission directly from the copyright holder. To view a copy of this licence, visit http://creativecommons.org/licenses/by/4.0/. 


\section{Introduction}

The use of mineral interfaces, in sand-sized rock fragments, has been determined for plutoniclastic sand in first-order stream sand (e.g., Heins 1993, 1995), glacial sand (e.g., Caracciolo et al. 2012), and in parent felsic plutonic and gneissic bedrock (e.g., Weltje et al. 2018). Heins $(1993,1995)$ demonstrated that the textural parameters of parent rock represent the main control on rock-fragment abundance in modern felsic plutoniclastic stream sands derived from granodioritic plutons of the Cordilleras of the United States and Mexico. These studies demonstrated that the textural parameters of the parent granodioritic source lithotypes represent the main control on rock-fragment abundance in modern plutoniclastic sand (Heins 1993), and that the types, and durability, of mineral interfaces, preserved inside the felsic sandy phanerites, are closely related to climate and topography of the source area (Heins 1995). Specifically, according to Heins (1995), some interfacial percentages correlate strongly with the environmental parameters of the weathering efficiency, such as slope (e.g., BA=Biotite/Accessory interface), drainage area (e.g., KK=K-feldspar/K-feldspar; $\mathrm{PlPl}=$ Plagioclase/Plagioclase interfaces), precipitation (e.g., $\mathrm{KB}=\mathrm{K}$-feldspar/Biotite interface), and temperature (e.g., $\mathrm{KO}=\mathrm{K}$-feldspar/Opaque interface). The interface analysis and the size-composition evolution of the rock fragment assemblage of the Swiss Alps plutoniclastic sand moraines (Caracciolo et al. 2012) indicate that, in terms of stability, $\mathrm{QQ}>\mathrm{PlPl}>\mathrm{QPl}>\mathrm{PlK}$ (where $\mathrm{Q}, \mathrm{Pl}$, and $\mathrm{K}$ stand for quartz, plagioclase, and $\mathrm{K}$ feldspar, respectively). These are the sandy-textured interfaces with the highest mechanical preservation potential, consistent with the assessment of Heins (1995) regarding the higher preservation potential of noisomineralic PIK and QK interfaces compared to isomineralic KK bonds. Weltje et al. (2018) parameterized crystal-interface frequencies in granitoids bedrock (granodiorite, granite, monzogranite and orthogneiss) outlining that a quantitative description of parent-rock texture has to be taken into account for regional sediment-generation studies.

Volcaniclastic epiclastic sandy grains consist of a mixture of chemically and mechanically durable felsitic grains such as rhyolite and dacite (e.g., Garzanti et al. 2002), with intermediate durability such as andesite (Mack and Jerzykiewicz 1989; Cather and Folk 1991; Smith and Lotosky 1995), and unstable such as basalts fragments (e.g., Marsaglia 1993; Critelli and Ingersoll 1995; Critelli et al. 2002; Morrone et al. 2017, 2020), and glass-rich hyaloclastite and pillow basalts grains (Furnes et al. 2008; Sætre et al. 2019). In this latter case, pores and vesicules diminish rapidly durability of pillow basalts and hyaloclastites source rock lithotypes (e.g., Haraldsson 1984; Fisk and McLoughlin 2013), with a consequent impoverishment of the sand fraction in the siliciclastic detritus (e.g., Palomares and Arribas 1993).

Deduction of interface properties from analysis of natural sediments is very often difficult (e.g., Weltje 2012; Weltje et al. 2018) and, even more for volcaniclastic lithic grains which are highly sensitive to chemical alteration (e.g., Marsaglia 1992; Johnsson et al. 1993; Le Pera and Morrone 2018) and to destruction by transport (Cameron and Blatt 1971; Davies et al. 1978; Cather and Folk 1991; Morrone et al. 2018) before the particles are buried by later pyroclastic events or reworked into the marine record (e.g., Morrone et al. 2017, 2020). The use of mineral interfaces in sand-sized rock fragments derived from effusive and pyroclastic source rocks has received much less attention, probably because they, especially their glassy component, are strongly dependent by chemical weathering (e.g., Johnsson et al. 1993), such as the very active and high rate of glass hydrolysis (e.g., White et al. 1986), glass breakdown and transformation to clay minerals (e.g., Lowe 1986; King 1986) or to both analcime and palagonite (e.g., Sætre et al. 2018, 2019).

To our knowledge, this is the first attempt to make a quantitative use of the volcaniclastic interfacial modal mineralogy of epiclastic sandy grains, to infer mechanical durability control of active reworking at beach environment sedimentation processes.

Modal mineralogy analysis on the types of mineral interfaces (e.g., Heins 1995), preserved in rock fragments of modern volcaniclastic Aeolian islands beach sand, has been carried out as a complementary research integrating, and further refining, existing petrographic analyses of both light framework grains (Critelli et al. 1993; Morrone et al. 2017, 2018) and heavy detrital minerals (Garzanti and Andò 2007a, 2007b) from an undissected volcanic arc setting sand (e.g., Dickinson 1985) such as the Aeolian magmatic arc.

The relationship between interfaces types, and amounts of changes, and shoreline transport mechanisms, can be useful to illustrate the effectiveness of high kinetic energy as a disintegrator of volcanic lithic sandy grains. Moreover, the proposed methodology could help to avoid underestimating or overestimating the contribution (or propensity to create sandy epiclastic detritus) supplied by volcanic source terranes in the stratigraphic record. This research documents the variability in texture before the particles are buried by later pyroclastic events or reworked into the marine record environment. The relationships existing into the conversion process such as "volcanic rock $\rightarrow$ volcaniclastic sand"-deduced from this actualistic study-might supply a useful methodological and research contribute to understanding provenance of ancient volcanogenic sequences in the stratigraphic record. The present study aims to demonstrate the advantages of: 
a) defining the most important mineral interfaces inside volcanic rock fragments as potential breakage surfaces;

b) quantifying the main interfaces within the grains from the three grain size fractions (very coarse, coarse and medium) of the beach sand samples by petrographic point-counting procedures;

c) analyzing variation in the content of specific interfaces between these three sand-size fractions.

Moreover, another purpose of this study is to characterize rate and style of comminution (if related to crystal breakdown or to abrasion) of volcanic phanerites to monocrystalline grains at a modern beach environment. Interfaces within sand-sized volcaniclastic grains are much more complicated than those of plutoniclastic composition (e.g., Heins 1993, 1995; Caracciolo et al. 2012; Weltje et al. 2018), because they involve not only contact between crystalline (ordered) phases, such as between minerals, but also more complex contacts between crystalline (ordered) and amorphous (disordered) phases interfaces such as contacts between a phenocrystal (ordered) and a glassy groundmass (disordered).

\section{Geological background and samples location}

The Aeolian archipelago, located between the southern Tyrrhenian Sea backarc basin and the Calabrian Arc forearc region, is composed of seven islands and submerged seamounts arranged approximately in a ring-like structure, at the boundary between the African and Eurasian plates (Fig. 1). The Aeolian Islands were emplaced on a thinned $\sim 20 \mathrm{~km}$ continental crust and so are considered examples of continental-arc volcanism (Barberi et al. 1974; Keller 1982; Ventura et al. 1999; Ventura 2013). The volcanic products, whose age ranges from 1.03 Ma to the present (Tranne et al. 2002a, 2002b; De Rosa et al. 2003; Lucchi 2009), belong to the calcalkaline, high-K calcalkaline, shoshonitic and alkaline potassic associations (Barberi et al. 1974; Keller 1982).

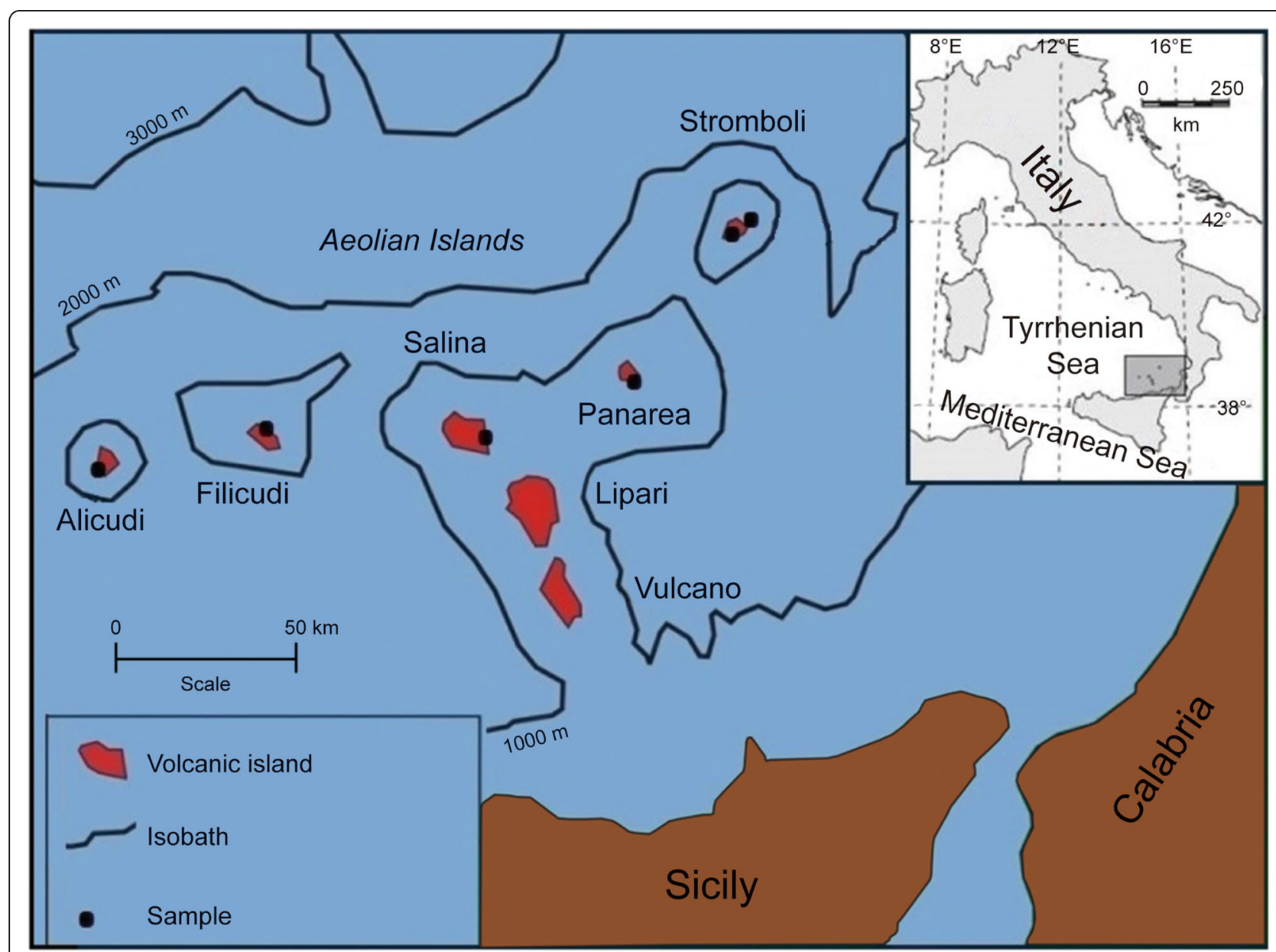

Fig. 1 Geological sketch map of the Aeolian archipelago and sand sample location (AL-3=Alicudi beach sand sample; Fi-3=Filicudi beach sand sample; SA-1 = Salina beach sand sample; PN-1 = Panarea beach sand sample; STR-1 = Stromboli beach sand sample; STR-9= Stromboli crateric sand sample) 
The geochemical affinity of these rocks, and the occurrence of deep seismicity (up to $550 \mathrm{~km}$ ) below the Southern Tyrrhenian Sea, has led many authors to relate the Aeolian volcanic arc to the SE rollback of the Ionian slab below the Calabrian Arc (e.g. Patacca and Scandone 1989; Faccenna et al. 2001; Gvirtzman and Nur 2001; Chiarabba et al. 2008).

Five beach sand samples were collected from high-tide berms spaced along Alicudi, Filicudi, Salina, Panarea and Stromboli islands accessible coastal perimeters; only one sand sample was collected near the crateric centre of Stromboli representing a first-order scale of sampling as defined by Ingersoll (1990) and later on by Critelli et al. (1997) (Fig. 1).

\subsection{Alicudi Island source rocks}

The quiescent Alicudi volcano is sited at the westernmost margin of the Aeolian archipelago (Fig. 1) and, its subaerial portion is made up predominantly of lava flows and domes and minor pyroclastic rocks (Lucchi et al. 2013a). The Alicudi main volcanic products include calc-alkaline basalts and basaltic andesites (Villari 1980; Peccerillo et al. 2013), erupted during early phases of activity (c. $106 \mathrm{ka}$ to $80 \mathrm{ka}$; Lucchi et al. 2013a), whereas andesites became dominant during latest stages of development of Alicudi (c. 80 ka to 28 ka; Lucchi et al. 2013a). All the rocks (lavas and pyroclastic rocks) from Alicudi Island are highly porphyritic. These rocks are basalts and basaltic andesites, dominated by plagioclase, clinopyroxene, minor olivine and brown hornblende phenocrysts, set in a glassy or a microlitic groundmass (e.g., Lucchi et al. 2013a). Phenocrysts mineralogy of andesites exhibits higher proportion of plagioclase with respect to pyroxenes, brown hornblende and olivine and accessory minerals such as $\mathrm{Fe}-\mathrm{Ti}$ oxides set in a microlitic groundmass with also some interstitial glass (e.g., Lucchi et al. 2013a).

\subsection{Filicudi Island source rocks}

Filicudi lavas and pyroclastic products compositions range from calc-alkaline basalts, basaltic andesites, high$\mathrm{K}$ andesites with minor dacites (e.g., Lucchi et al. 2013b). The oldest exposed products are prevalently basalts and basaltic andesites (c. $246 \mathrm{ka}$ to $146 \mathrm{ka}$ ) (Paleo-Filicudi sensu Lucchi et al. 2013b); younger rocks are high-K andesites and dacites (c. 64 ka) (e.g., Lucchi et al. 2013b). All these rocks are highly porphyritic: phenocrysts mineralogy of basalts and basaltic andesites consists of plagioclase, pyroxenes, olivine and the groundmass between minerals is glassy or microcrystalline. Plagioclase phenocrystals are the dominant mineral phases in the high- $\mathrm{K}$ andesites and dacites which also contain small amounts of pyroxenes, biotite and brown hornblende. Ti-magnetite, ilmenite and apatite are accessory minerals and the groundmass generally contains the same mineral phases as the phenocrysts as microphenocrysts or glassy material (e.g., Lucchi et al. 2013b).

\subsection{Salina Island source rocks}

Salina Island is located in the center of the Aeolian archipelago (Fig. 1) and it is considered a crucial sector of the entire geodynamic framework of the Aeolian region (Lucchi et al. 2013c). According to Lucchi et al. (2013c), the volcanic rocks of Salina, have a variable range in composition, from basalts to basaltic andesites, andesites, dacites and rhyolites lavas and pyroclastic rocks. Basalt is by far the most abundant rock type on Salina whereas dacites and rhyolites are subordinate in volume. Basaltic and basaltic andesite lava flows are the oldest ( $c$. 244-160 ka) Salina rocks whereas andesitic, dacitic and rhyolitic lavas were generated during the final stages of the volcanic activity of the island (c. 121-15.6 ka) (Lucchi et al. 2013c). Phenocrystals and groundmass mineralogy of Salina basalts, basaltic andesites and andesites is similar to that of Alicudi and Filicudi described previously. Dacites contain phenocrystals of pyroxenes, amphibole enclosed within a microlitic to vitric groundmass and rhyolitic pumices are characterized by plagioclase, amphibole and biotite, less abundant pyroxenes and olivine in a vesicular glassy groundmass (e.g., Lucchi et al. 2013c).

\subsection{Panarea Island source rocks}

The Panarea volcanic rocks range from mafic to felsic; dacites and high- $\mathrm{K}$ dacites are the most common rock types, followed by andesites, and minor basalts (Lucchi et al. 2013d). The island is a dome-field almost entirely constructed between $c .155-149 \mathrm{ka}$ and $c .118 \mathrm{ka}$ by a sequence of andesitic to dacitic lava domes and lava flows and subordinate pyroclastic products (Lucchi et al. 2013d). Plagioclase is the dominant phenocrystic phase for all the rocks, followed by pyroxenes. Amphibole, often transformed to opaque minerals, appears as phenocryst in the andesites and becomes more abundant in the dacites. Biotite occurs as a phenocryst in the rhyolites and basalts. These are poorly porphyritic and vesicular, have phenocrysts of plagioclase and pyroxene, surrounded by a dark glassy groundmass. Rhyolites have a glassy, fluidal groundmass, and dacites have banded groundmass given by an heterogeneous distribution of microliths (Lucchi et al. 2013d).

\subsection{Stromboli Island source rocks}

The Stromboli Island is located in the northeasternmost portion of the Aeolian archipelago (Fig. 1) with its subaerial part mainly built up during the last $c$. 85 ka (e.g., Francalanci et al. 2013). Stromboli volcano shows one of the largest rock compositional variations 
in the Aeolian Islands: detailed field mapping, combined with petrological and geochemical studies (Keller et al. 1993; Hornig-Kjarsgaard et al. 1993), allowed reconstruction of main cycles of volcanic activity subdivided into cycles defined as a Paleostromboli $(<100-13 \mathrm{ka})$, a Neostromboli (13-6 ka) and a Recent Stromboli $(<6 \mathrm{ka})$ (e.g., Francalanci et al. 2013). In terms of compositional features of the erupted products, they range from basalts, basaltic andesites and andesite for Paleostromboli, shoshonites, latites and trachytes for Neostromboli and the most recent products of Recent Stromboli are shifted towards basaltic composition (e.g., Francalanci et al. 2013). These rocks generally have seriate porphyritic textures with variable phenocrystals content (c. 5-55 vol\%). Plagioclase is the most abundant phenocryst and microphenocryst of the basalts, followed by pyroxenes, olivine and small amounts of hornblende, biotite, leucite and opaques. Latites are characterized by glassy groundmass and K-feldspar, phlogopite and biotite are present in the bulk-rock groundmass of the high-K lavas (Hornig-Kjarsgaard et al. 1993).

\section{Methods}

The five sand samples from beach environment, and the only one crateric sand sample, were subsampled using a splitter, then washed using $\mathrm{H}_{2} \mathrm{O}_{2}$ to remove clay minerals and organic matter, air-dried and mechanically sieved using $1 \Phi$ intervals. Three size fractions of sand (2.00-1.00 mm, 0.50-0.25 mm and 0.25-0.125 mm), impregnated with epoxy, were then used to prepare 18 thin sections. Thin sections were subsequently stained for both calcium-rich and potassium-rich feldspar grains according to the method outlined in Marsaglia and Tazaki (1992). These size fractions were chosen because they are the most likely to be richer in polycrystalline polymineralic phaneritic rock fragments, with respect to finer size fractions, where monomineralic grains dominate the clastic assemblages (e.g., Pettijohn et al., 1987; Ingersoll et al. 1984). Petrographic data of analyzed sands include counts to determine interface types, frequency, and polycrystallinity in polymineralic phaneritic volcanic rock fragments of the Aeolian beach modern sand. Analyzed and counted interfaces are contained within phaneritic volcanic grains with lathwork texture ( $L v l$ of Marsaglia 1992, 1993) and seriate porphyritic texture, this latter textural category not included within Marsaglia's classification scheme of volcaniclastic sandy particles. Aeolian islands beaches sandy grains, with this latter volcanic texture, contain commonly aggregated phenocrystals of pyroxene or olivine, referring to as glomerocrystals embedded in a microlitic groundmass (e.g., Morrone et al. 2017). In some cases, such glomerocrystals are aggregates of isomineralic and isostructural mineral phases (e.g., sensu Heins 1995), so an isomineralic interface has been counted; more commonly they are composed of two or more minerals, and then a no-isomineralic interface has been counted (Table 1, Fig. 2). The phenocrystals adjoining with a glassy groundmass define interfaces such as the $\mathrm{Pl} / \mathrm{Glgr}$ (Plagioclase/Glassy groundmass) interface, those phenocrystal adjoining with a microlitic groundmass define interfaces such as $\mathrm{Pl} /$ Migr (Plagioclase/Microlitic groundmass), and other formal categories used to tabulate interfacial counts shown in Table 1. Isomineralic interfaces are those between phenocrystals with identical chemistry and structure such as $\mathrm{Ol} / \mathrm{Ol}$ (Olivine/Olivine), Op/Op (Opaque/Opaque), etc., for which the initials are also shown in Table 1. Counted mineral-mineral interfaces, mineral-glassy groundmass and mineral/microlitic groundmass interfaces of the volcanic rock fragments, contained in the very coarse, coarse and medium grain size, were assigned to the categories listed in Table 1 . For 100 volcanic lithic grains contained in each of the 18 analyzed thin sections of the sands, the number of counted interface ranges from a minimum value of 872 (Fi-3 m sample of Filicudi island beach) to a maximum of 5941 interfaces (SA-1-Vc sample of Salina Island beach) (Tables 2, 3 and 4). The petrographic analysis of the thin sections was used to determine the exact nature of the crystal/crystal interfaces, of the crystals/glassy groundmass interfaces, and of the crystals/microlitic groundmass explained in Table 1 , crucial because the interface is the boundary where breakdown process occurs (e.g., Cather and Folk 1991; Heins 1993, 1995; Palomares and Arribas 1993; Caracciolo et al. 2012; Weltje et al. 2018). For measurement procedure the interfaces types of Table 1, each thin section has been moved, using a mechanical stage, taking into account the interfaces types contained within 100 volcanic grains on a $28 \times 28 \mathrm{~mm}$ coverage area. On each of the $100 \mathrm{vol}-$ canic grains, each interface between a crystal and the glassy, or microlitic, groundmasses, and between isomineralic or no-isomineralic crystals have been identified and counted under the polarizing microscope (Tables 1, 2, 3 and 4; Figs. 7, 8, 9 and 10).

\section{Results of modal mineralogy data}

\subsection{Alicudi Island (AL-3)}

Interface types range between 28 and 26 categories with a total of 2725 in the very coarse sand, 2319 in the coarse sand and 1662 in the medium sand counted categories, respectively (Table 2). The interfacial composition of the very coarse, coarse and medium sand is quite similar: $\mathrm{Pl} / \mathrm{Glgr}$ (Plagioclase/Glassy groundmass), $\mathrm{Px} / \mathrm{Glgr}$ (Pyroxene/Glassy groundmass) and Ol/Glgr (Olivine/ Glassy groundmass) are the three interfacial heterogeneous types dominating among other less abundant interfacial types (Table 2 and Fig. 3). The coarse 
Table 1 Point-counting categories of interfaces. Interfaces described using combination of the letters defined below

\begin{tabular}{ll}
\hline No-isostructural interfaces & Glass-rich interfaces \\
1. Op/Glgr & Opaque/Glassy groundmass \\
2. Pl/Glgr & Plagioclase/Glassy groundmass \\
3. Px/Glgr & Pyroxene/ Glassy groundmass \\
4. Ol/Glgr & Olivine/ Glassy groundmass \\
5. Hbl/Glgr & Hornblende/Glassy groundmass \\
6. Idd/Glgr & Iddingsite/Glassy groundmass \\
7. Bt/Glgr & Biotite/Glassy groundmass \\
8. Rt/Glgr & Rutile/Glassy groundmass \\
& Glass-poor interfaces \\
1. Pl/Migr & Plagioclase/Microlitic groundmass \\
2. Px/Migr & Pyroxene/Microlitic groundmass \\
3. Op/Migr & Opaque/Microlitic groundmass \\
4. Ol/Migr & Olivine/Microlitic groundmass \\
5. Hbl/Migr & Hornblende/Microlitic groundmass \\
6. Rt/Migr & Rutile/Microlitic groundmass \\
7. Bt/Migr & Biotite/Microlitic groundmass \\
\hline
\end{tabular}

No-isomineralic interfaces

\begin{tabular}{|c|c|}
\hline 1. Op/Ol & Opaque/Olivine \\
\hline 2. $\mathrm{Px} / \mathrm{Ol}$ & Pyroxene/Olivine \\
\hline 3. $\mathrm{Pl} / \mathrm{Ol}$ & Plagioclase/Olivine \\
\hline 4. PI/Op & Plagioclase/Opaque \\
\hline 5. Px/Op & Pyroxene/Opaque \\
\hline 6. $\mathrm{Pl} / \mathrm{Px}$ & Plagioclase/Pyroxene \\
\hline 7. Ol/ldd & Olivine/Iddingsite \\
\hline 8. $\mathrm{Hbl} / \mathrm{Pl}$ & Hornblende/Plagioclase \\
\hline 9. $\mathrm{Hbl} / \mathrm{Px}$ & Hornblende/Pyroxene \\
\hline 10. Bt/P & Biotite/Plagioclase \\
\hline 11. Bt/Px & Biotite/Pyroxene \\
\hline 12. Bt/Op & Biotite/Opaque \\
\hline 13. $\mathrm{Hbl} / \mathrm{Op}$ & Hornblende/Opaque \\
\hline 14. $\mathrm{ldd} / \mathrm{Px}$ & Iddingsite/Pyroxene \\
\hline 15. Idd/PI & Iddingsite/Plagioclase \\
\hline 16. Ol/Bt & Olivine/Biotite \\
\hline 17.Ol/Hbl & Olivine/Hornblende \\
\hline 18. $\mathrm{Bt} / \mathrm{Hbl}$ & Biotite/Hornblende \\
\hline \multicolumn{2}{|c|}{ Iso-mineralic interfaces } \\
\hline 1. $\mathrm{Pl} / \mathrm{PI}$ & Plagioclase/Plagioclase \\
\hline 2. $\mathrm{Px} / \mathrm{Px}$ & Pyroxene/Pyroxene \\
\hline 3. Ol/OI & Olivine/Olivine \\
\hline 4. Op/Op & Opaque/Opaque \\
\hline 5. $\mathrm{Hbl} / \mathrm{Hbl}$ & Hornblende/Hornblende \\
\hline 6. $\mathrm{Bt} / \mathrm{Bt}$ & Biotite/Biotite \\
\hline
\end{tabular}

sand is the grain-size with the highest number of interfaces typologies followed by medium and very coarse sand grain-sizes (Table 2). Compositional variation, regardless of interface types composition, within the sand of Alicudi beach, is accompanied by interfacial percentage decreasing with decreasing grain size: the conversion from very coarse to coarse sand led to a percentage decrease, in all of the interface types, equal to $6.0 \%$ and to $10.0 \%$ from the coarse to the medium grain size, respectively (Fig. 6a). Cumulatively, the conversion from very coarse to medium sand grain size is related to a decrease of $16.0 \%$ of the overall interfaces types. The most abundant interfaces type of the Alicudi Island beach sand, the no-isostructural interface plagioclase/glassy groundmass (Pl/Glgr interface of Table 1) shows a decreasing trend with decreasing grain-size and with an average decreasing of at least $10 \%$ (Fig. $6 \mathrm{~g}$ ) from the very coarse $(43.03 \%)$ to the coarse $(33.90 \%)$ up to the medium (23.06\%) sand fraction. Conversely, the no-isostructural hornblende/glassy groundmass ( $\mathrm{Hbl} / \mathrm{Glgr}$ interface of Table 1) is characterized by a trend reverse to that of the $\mathrm{Pl} / \mathrm{Glgr}$ interface with an evident increase of its abundance with decreasing grain size of the sand (Fig. $6 \mathrm{~g}$ ), probably due to the hornblende double system of cleavage angles that significantly controls disintegration of its crystal habit (e.g., Velbel 1989; Heins 1995).

\subsection{Filicudi Island (Fi-3)}

Interface types range between 27 and 33 categories with a total of 3118 in the very coarse sand, 2262 in the coarse sand and 872 in the medium sand counted categories, respectively (Table 2). As in Alicudi Island sand, the interfacial composition of the very coarse, coarse and medium sand is quite similar: Pl/Glgr (Plagioclase/ Glassy groundmass), Px/Glgr (Pyroxene/Glassy groundmass) and $\mathrm{Px} / \mathrm{Op}$ (Pyroxene/Opaque), are the three interfacial heterogeneous, or no-isostructural types dominating among other less abundant interfacial types (Table 2 and Fig. 3). The overall interfacial percentage decrease, with decreasing grain size, and it is equal to $9.07 \%$ during the conversion from very coarse to coarse sand and to $23.43 \%$ from coarse to medium sand. The conversion from very coarse to medium sand grain size is related to a decrease of $32.5 \%$ of the overall interface types (Fig. 6b). In Filicudi Island beach, very coarse sand is characterized mainly by no-isomineralic interfaces such as the Px/Op (Pyroxene/Opaque interface) (Fig. $6 \mathrm{~h})$, considered one of the interfaces with the lowest mechanical durability during transport (e.g., Heins 1995; Caracciolo et al. 2012; Weltje et al. 2018). During the conversion from very coarse sand to medium sand size, $\mathrm{Px} / \mathrm{Op}$ interfaces records an average decrease of at least $40 \%$ and in the amount of $10 \%$ during the conversion from coarse to medium sand (Fig. 6h). The overall Px/ 


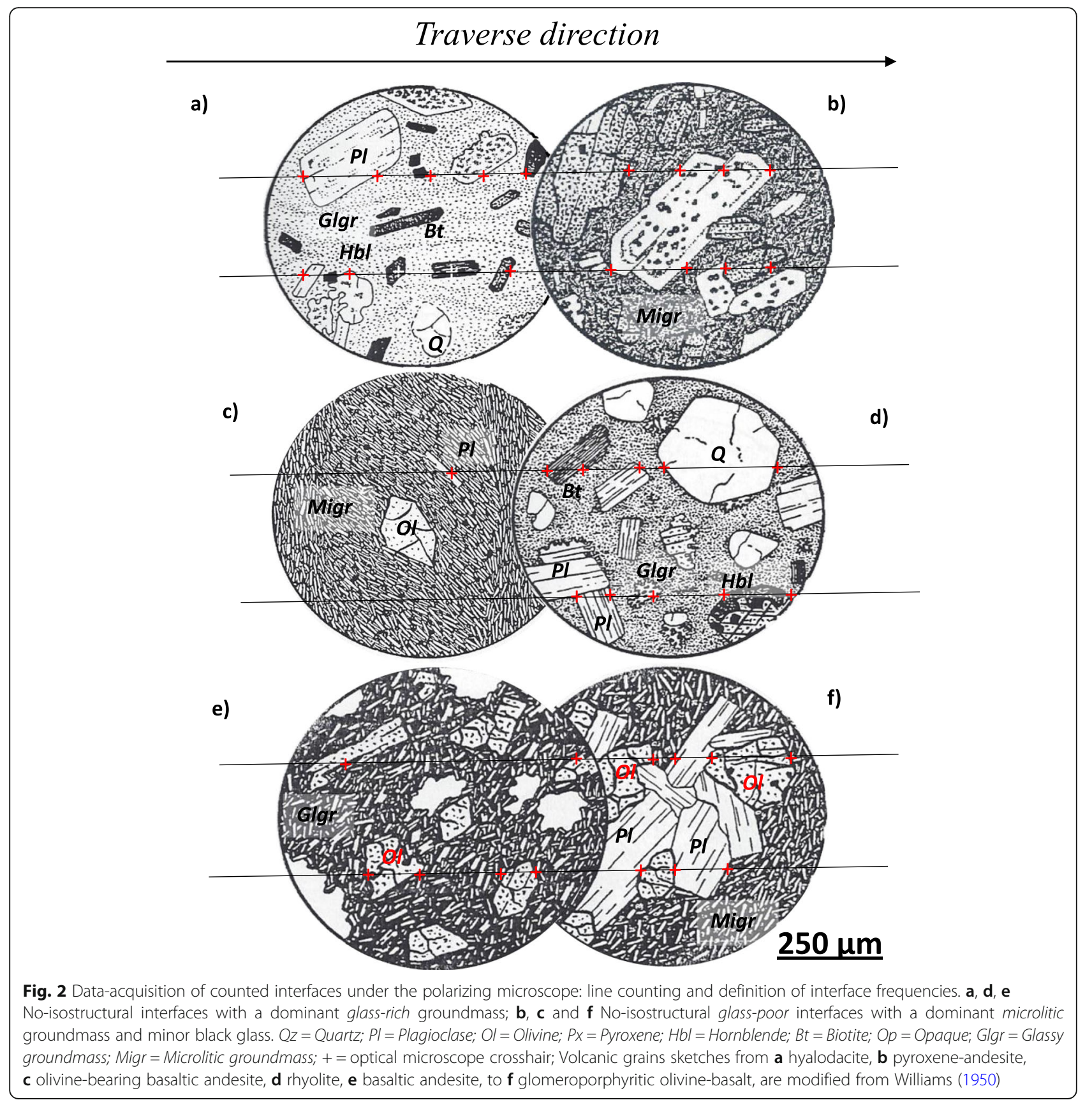

Op interface decrease, from the very coarse to the medium sand, is equal to $57 \%$, with this very high reduction due to this sharp heterophase interfaces (e.g., Balluffi et al. 2005), separating two different domains with different crystal lattices such as a single-chain inosilicate (Pyroxene) and an opaque mineral (Op). This interface, located along this plane, characterized by weak atomic bonds, will be broken generally faster than other interfaces (e.g., Eggleton 1986; Heins 1995). Another noisomineralic interface, such as the $\mathrm{Pl} / \mathrm{Py}$ (Plagioclase/Pyroxene interface) (Fig. 6h, Table 1), reveals an interfaces loss nearly similar to the Px/Op interface decreasing trend: in this case a no-isostructural boundary, between a tectosilicate (Plagioclase) and a single-chain silicate (Pyroxene), will enhance the destruction of the primary polymineralic grain (Fig. 6h). Moreover, intra-crystals twinning and compositional zoning of both plagioclase (e.g., Slatt and Eyles 1981; Garzanti 1986; Heins 1995) and pyroxene (e.g., Davies et al. 1978; Morrone et al. 2017) will amplify grain shattering. $\mathrm{Pl} / \mathrm{Py}$ interface decreases $34.0 \%$ during the transition from very coarse to coarse sand and $16 \%$ from coarse to medium sand size; 
Table 2 Interface types results from a point counting of 100 grains from very coarse $(1-2 \mathrm{~mm})$, coarse (1-0.5 mm) and medium $(0.5-0.25 \mathrm{~mm}$ ) grain size fractions from Alicudi and Filicudi sand samples (AL = Alicudi Island; Fi = Filicudi Island; Vc=very coarse, $C=$ coarse, $\mathrm{m}=$ medium)

\begin{tabular}{|c|c|c|c|c|c|c|c|c|c|c|c|c|}
\hline \multirow{2}{*}{$\begin{array}{l}\text { Interface } \\
\text { type }\end{array}$} & \multicolumn{2}{|c|}{$A L-3-V c$} & \multicolumn{2}{|c|}{$A L-3-C$} & \multicolumn{2}{|c|}{$A L-3-m$} & \multicolumn{2}{|c|}{ Fi-3-Vc } & \multicolumn{2}{|l|}{$F i-3-C$} & \multicolumn{2}{|c|}{$F i-3-m$} \\
\hline & $\mathrm{N}$. & $\%$ & $\mathrm{~N}$. & $\%$ & $\mathrm{~N}$. & $\%$ & $\mathrm{~N}$. & $\%$ & $\mathrm{~N}$. & $\%$ & $\mathrm{~N}$. & $\%$ \\
\hline Op/Glgr & 63 & 2.31 & 49 & 2.11 & 101 & 6.08 & 155 & 4.97 & 90 & 3.98 & 63 & 7.22 \\
\hline $\mathrm{Pl} / \mathrm{Glgr}$ & 1127 & 41.36 & 888 & 38.29 & 604 & 36.34 & 911 & 29.22 & 764 & 33.78 & 249 & 28.56 \\
\hline Px/Glgr & 451 & 16.55 & 327 & 14.10 & 236 & 14.20 & 373 & 11.96 & 265 & 11.72 & 95 & 10.89 \\
\hline $\mathrm{Ol} / \mathrm{Glgr}$ & 304 & 11.16 & 253 & 10.91 & 141 & 8.48 & 96 & 3.08 & 88 & 3.89 & 63 & 7.22 \\
\hline $\mathrm{Hbl} / \mathrm{Glgr}$ & 40 & 1.47 & 63 & 2.72 & 83 & 4.99 & 63 & 2.02 & 106 & 4.69 & 26 & 2.98 \\
\hline Idd/Glgr & 5 & 0.18 & 13 & 0.56 & 0 & 0.00 & 6 & 0.19 & 0 & 0.00 & 0 & 0.00 \\
\hline $\mathrm{Bt} / \mathrm{Glgr}$ & 5 & 0.18 & 0 & 0.00 & 0 & 0.00 & 57 & 1.83 & 30 & 1.33 & 0 & 0.00 \\
\hline $\mathrm{Rt} / \mathrm{Glgr}$ & 0 & 0.00 & 0 & 0.00 & 0 & 0.00 & 0 & 0.00 & 0 & 0.00 & 0 & 0.00 \\
\hline $\mathrm{Pl} / \mathrm{Migr}$ & 95 & 3.49 & 86 & 3.71 & 28 & 1.68 & 148 & 4.75 & 78 & 3.45 & 11 & 1.26 \\
\hline Px/Migr & 28 & 1.03 & 24 & 1.03 & 5 & 0.30 & 73 & 2.34 & 41 & 1.81 & 0 & 0.00 \\
\hline Op/Migr & 28 & 1.03 & 50 & 2.16 & 21 & 1.26 & 114 & 3.66 & 95 & 4.20 & 0 & 0.00 \\
\hline Ol/Migr & 5 & 0.18 & 0 & 0.00 & 8 & 0.48 & 34 & 1.09 & 5 & 0.22 & 9 & 1.03 \\
\hline $\mathrm{Hbl} / \mathrm{Migr}$ & 0 & 0.00 & 0 & 0.00 & 3 & 0.18 & 4 & 0.13 & 7.00 & 0.31 & 0 & 0.00 \\
\hline Rt/Migr & 0 & 0.00 & 0 & 0.00 & 0 & 0.00 & 0 & 0.00 & 0 & 0.00 & 0 & 0.00 \\
\hline Bt/Migr & 0 & 0.00 & 0 & 0.00 & 0 & 0.00 & 0 & 0.00 & 0 & 0.00 & 14 & 1.61 \\
\hline Op/OI & 46 & 1.69 & 87 & 3.75 & 53 & 3.19 & 42 & 1.35 & 56 & 2.48 & 27 & 3.10 \\
\hline $\mathrm{Px} / \mathrm{Ol}$ & 53 & 1.94 & 20 & 0.86 & 19 & 1.14 & 10 & 0.32 & 15 & 0.66 & 9 & 1.03 \\
\hline $\mathrm{Pl} / \mathrm{Ol}$ & 40 & 1.47 & 37 & 1.60 & 31 & 1.87 & 53 & 1.70 & 34 & 1.50 & 11 & 1.26 \\
\hline $\mathrm{Pl} / \mathrm{Op}$ & 56 & 2.06 & 71 & 3.06 & 17 & 1.02 & 25 & 0.80 & 117 & 5.17 & 19 & 2.18 \\
\hline Px/Op & 65 & 2.39 & 64 & 2.76 & 50 & 3.01 & 308 & 9.88 & 129 & 5.70 & 39 & 4.47 \\
\hline $\mathrm{Pl} / \mathrm{Px}$ & 66 & 2.42 & 51 & 2.20 & 31 & 1.87 & 167 & 5.36 & 75 & 3.32 & 31 & 3.56 \\
\hline $\mathrm{Ol} / \mathrm{ldd}$ & 15 & 0.55 & 21 & 0.91 & 13 & 0.78 & 15 & 0.48 & 3 & 0.13 & 9 & 1.03 \\
\hline $\mathrm{Hbl} / \mathrm{Pl}$ & 13 & 0.48 & 13 & 0.56 & 23 & 1.38 & 35 & 1.12 & 35 & 1.55 & 3 & 0.34 \\
\hline $\mathrm{Hbl} / \mathrm{Px}$ & 10 & 0.37 & 1 & 0.04 & 4 & 0.24 & 8 & 0.26 & 8 & 0.35 & 3 & 0.34 \\
\hline $\mathrm{Bt} / \mathrm{Pl}$ & 0 & 0.00 & 0 & 0.00 & 0 & 0.00 & 8 & 0.26 & 9 & 0.40 & 6 & 0.69 \\
\hline $\mathrm{Bt} / \mathrm{Px}$ & 0 & 0.00 & 0 & 0.00 & 0 & 0.00 & 16 & 0.51 & 2 & 0.09 & 1 & 0.11 \\
\hline $\mathrm{Bt} / \mathrm{Op}$ & 0 & 0.00 & 25 & 1.08 & 0 & 0.00 & 28 & 0.90 & 4 & 0.18 & 2 & 0.23 \\
\hline $\mathrm{Hbl} / \mathrm{Op}$ & 16 & 0.59 & 25 & 1.08 & 48 & 2.89 & 90 & 2.89 & 72 & 3.18 & 48 & 5.50 \\
\hline Idd/Px & 5 & 0.18 & 3 & 0.13 & 0 & 0.00 & 6 & 0.19 & 0 & 0.00 & 0 & 0.00 \\
\hline$|d d / P|$ & 1 & 0.04 & 1 & 0.04 & 6 & 0.36 & 0 & 0.00 & 0 & 0.00 & 0 & 0.00 \\
\hline $\mathrm{Ol} / \mathrm{Bt}$ & 0 & 0.00 & 0 & 0.00 & 0 & 0.00 & 7 & 0.22 & 0 & 0.00 & 0 & 0.00 \\
\hline $\mathrm{Ol} / \mathrm{Hbl}$ & 0 & 0.00 & 5 & 0.00 & 5 & 0.30 & 0 & 0.00 & 3 & 0.13 & 0 & 0.00 \\
\hline $\mathrm{Bt} / \mathrm{Hbl}$ & 0 & 0.00 & 0 & 0.22 & 0 & 0.00 & 0 & 0.00 & 0 & 0.00 & 0 & 0.00 \\
\hline $\mathrm{Pl} / \mathrm{PI}$ & 117 & 4.29 & 108 & 4.66 & 86 & 5.17 & 160 & 5.13 & 95 & 4.20 & 88 & 10.09 \\
\hline $\mathrm{Px} / \mathrm{Px}$ & 25 & 0.92 & 14 & 0.60 & 13 & 0.78 & 52 & 1.67 & 18 & 0.80 & 26 & 2.98 \\
\hline $\mathrm{Ol} / \mathrm{Ol}$ & 46 & 1.69 & 18 & 0.78 & 15 & 0.90 & 32 & 1.03 & 8 & 0.35 & 11 & 1.26 \\
\hline Op/Op & 0 & 0.00 & 1 & 0.04 & 8 & 0.48 & 12 & 0.38 & 4 & 0.18 & 6 & 0.69 \\
\hline $\mathrm{Hbl} / \mathrm{Hbl}$ & 0 & 0.00 & 1 & 0.04 & 10 & 0.60 & 9 & 0.29 & 6 & 0.27 & 2 & 0.23 \\
\hline $\mathrm{Bt} / \mathrm{Bt}$ & 0 & 0.00 & 0 & 0.00 & 0 & 0.00 & 1 & 0.03 & 0 & 0.00 & 1 & 0.11 \\
\hline TOT & 2725 & 100 & 2319 & 100 & 1662 & 100 & 3118 & 100 & 2262 & 100 & 872 & 100 \\
\hline
\end{tabular}


Table 3 Interface types results from a point counting of 100 grains from very coarse $(1-2 \mathrm{~mm})$, coarse (1-0.5 mm) and medium $(0.5-0.25 \mathrm{~mm}$ ) grain size fractions from Panarea and Salina sand samples (PN= Panarea Island; SA = Salina Island; Vc=very coarse, $\mathrm{C}=$ coarse, $\mathrm{m}=$ medium)

\begin{tabular}{|c|c|c|c|c|c|c|c|c|c|c|c|c|}
\hline \multirow{2}{*}{$\begin{array}{l}\text { Interface } \\
\text { type }\end{array}$} & \multicolumn{2}{|c|}{$P N-1-V c$} & \multicolumn{2}{|c|}{$P N-1-C$} & \multicolumn{2}{|c|}{$P N-1-m$} & \multicolumn{2}{|c|}{$S A-1-V c$} & \multicolumn{2}{|c|}{$S A-1-C$} & \multicolumn{2}{|c|}{$S A-1-m$} \\
\hline & $\mathrm{N}$. & $\%$ & $\mathrm{~N}$. & $\%$ & $\overline{\mathrm{N}}$ & $\%$ & $\mathrm{~N}$. & $\%$ & $\mathrm{~N}$. & $\%$ & $\mathrm{~N}$. & $\%$ \\
\hline $\mathrm{Op} / \mathrm{Glgr}$ & 572 & 14.99 & 294 & 9.67 & 171 & 13.56 & 285 & 4.80 & 232 & 5.46 & 130 & 4.78 \\
\hline $\mathrm{Pl} / \mathrm{Glgr}$ & 1151 & 30.15 & 790 & 26.00 & 400 & 31.72 & 2347 & 39.51 & 1613 & 37.98 & 929 & 34.18 \\
\hline $\mathrm{Px} / \mathrm{Glgr}$ & 122 & 3.20 & 102 & 3.36 & 76 & 6.03 & 661 & 11.13 & 692 & 16.29 & 328 & 12.07 \\
\hline $\mathrm{Ol} / \mathrm{Glgr}$ & 37 & 0.97 & 21 & 0.69 & 18 & 1.43 & 118 & 1.99 & 93 & 2.19 & 73 & 2.69 \\
\hline $\mathrm{Hbl} / \mathrm{Glgr}$ & 377 & 9.88 & 333 & 10.96 & 130 & 10.31 & 68 & 1.14 & 4 & 0.09 & 26 & 0.96 \\
\hline Idd/Glgr & 0 & 0.00 & 0 & 0.00 & 0 & 0.00 & 20 & 0.34 & 0 & 0.00 & 0 & 0.00 \\
\hline $\mathrm{Bt} / \mathrm{Glgr}$ & 141 & 3.69 & 31 & 1.02 & 18 & 1.43 & 2 & 0.03 & 5 & 0.12 & 10 & 0.37 \\
\hline $\mathrm{Rt} / \mathrm{Glgr}$ & 5 & 0.13 & 0 & 0.00 & 0 & 0.00 & 0 & 0.00 & 0 & 0.00 & 0 & 0.00 \\
\hline $\mathrm{Pl} / \mathrm{Migr}$ & 159 & 4.17 & 289 & 9.51 & 4 & 0.32 & 623 & 10.49 & 369 & 8.69 & 250 & 9.20 \\
\hline Px/Migr & 49 & 1.28 & 11 & 0.36 & 1 & 0.08 & 154 & 2.59 & 219 & 5.16 & 105 & 3.86 \\
\hline Op/Migr & 75 & 1.96 & 117 & 3.85 & 3 & 0.24 & 132 & 2.22 & 246 & 5.79 & 126 & 4.64 \\
\hline Ol/Migr & 0 & 0.00 & 0 & 0.00 & 0 & 0.00 & 27 & 0.45 & 0 & 0.00 & 0 & 0.00 \\
\hline $\mathrm{Hbl} / \mathrm{Migr}$ & 6 & 0.16 & 69 & 2.27 & 6 & 0.48 & 0 & 0.00 & 0.00 & 0.00 & 0 & 0.00 \\
\hline Rt/Migr & 5 & 0.13 & 0 & 0.00 & 0 & 0.00 & 0 & 0.00 & 0 & 0.00 & 0 & 0.00 \\
\hline Bt/Migr & 0 & 0.00 & 7 & 0.23 & 0 & 0.00 & 0 & 0.00 & 0 & 0.00 & 5 & 0.18 \\
\hline Op/OI & 0 & 0.00 & 16 & 0.53 & 0 & 0.00 & 2 & 0.03 & 0 & 0.00 & 26 & 0.96 \\
\hline $\mathrm{Px} / \mathrm{Ol}$ & 10 & 0.26 & 1 & 0.03 & 3 & 0.24 & 83 & 1.40 & 13 & 0.31 & 2 & 0.07 \\
\hline $\mathrm{Pl} / \mathrm{Ol}$ & 15 & 0.39 & 82 & 2.70 & 3 & 0.24 & 30 & 0.50 & 10 & 0.24 & 18 & 0.66 \\
\hline PI/Op & 237 & 6.21 & 0 & 0.00 & 31 & 2.46 & 155 & 2.61 & 147 & 3.46 & 189 & 6.95 \\
\hline Px/Op & 34 & 0.89 & 40 & 1.32 & 67 & 5.31 & 249 & 4.19 & 112 & 2.64 & 104 & 3.83 \\
\hline $\mathrm{Pl} / \mathrm{Px}$ & 30 & 0.79 & 4 & 0.13 & 15 & 1.19 & 234 & 3.94 & 148 & 3.48 & 89 & 3.27 \\
\hline $\mathrm{Ol} / \mathrm{ldd}$ & 0 & 0.00 & 0 & 0.00 & 0 & 0.00 & 11 & 0.19 & 12 & 0.28 & 0 & 0.00 \\
\hline $\mathrm{Hbl} / \mathrm{Pl}$ & 135 & 3.54 & 225 & 7.40 & 57 & 4.52 & 16 & 0.27 & 0 & 0.00 & 8 & 0.29 \\
\hline $\mathrm{Hbl} / \mathrm{Px}$ & 2 & 0.05 & 0 & 0.00 & 0 & 0.00 & 0 & 0.00 & 0 & 0.00 & 0 & 0.00 \\
\hline $\mathrm{Bt} / \mathrm{PI}$ & 36 & 0.94 & 19 & 0.63 & 6 & 0.48 & 2 & 0.03 & 0 & 0.00 & 0 & 0.00 \\
\hline $\mathrm{Bt} / \mathrm{Px}$ & 2 & 0.05 & 0 & 0.00 & 0 & 0.00 & 0 & 0.00 & 0 & 0.00 & 5 & 0.18 \\
\hline $\mathrm{Bt} / \mathrm{Op}$ & 145 & 3.80 & 12 & 0.39 & 22 & 1.74 & 0 & 0.00 & 1 & 0.02 & 8 & 0.29 \\
\hline $\mathrm{Hbl} / \mathrm{Op}$ & 184 & 4.82 & 347 & 11.42 & 167 & 13.24 & 0 & 0.00 & 0 & 0.00 & 35 & 1.29 \\
\hline Idd/Px & 0 & 0.00 & 0 & 0.00 & 0 & 0.00 & 0 & 0.00 & 0 & 0.00 & 0 & 0.00 \\
\hline$|d d / P|$ & 0 & 0.00 & 0 & 0.00 & 0 & 0.00 & 0 & 0.00 & 0 & 0.00 & 0 & 0.00 \\
\hline $\mathrm{Ol} / \mathrm{Bt}$ & 0 & 0.00 & 0 & 0.00 & 0 & 0.00 & 0 & 0.00 & 0 & 0.00 & 0 & 0.00 \\
\hline $\mathrm{Ol} / \mathrm{Hbl}$ & 0 & 0.00 & 10 & 0.33 & 0 & 0.00 & 0 & 0.00 & 0 & 0.00 & 0 & 0.00 \\
\hline $\mathrm{Bt} / \mathrm{Hbl}$ & 21 & 0.55 & 23 & 0.76 & 3 & 0.24 & 0 & 0.00 & 0 & 0.00 & 0 & 0.00 \\
\hline $\mathrm{Pl} / \mathrm{PI}$ & 200 & 5.24 & 143 & 4.71 & 41 & 3.25 & 580 & 9.76 & 202 & 4.76 & 184 & 6.77 \\
\hline $\mathrm{Px} / \mathrm{Px}$ & 29 & 0.76 & 22 & 0.72 & 8 & 0.63 & 121 & 2.04 & 106 & 2.50 & 65 & 2.39 \\
\hline $\mathrm{Ol} / \mathrm{Ol}$ & 8 & 0.21 & 0 & 0.00 & 2 & 0.16 & 9 & 0.15 & 11 & 0.26 & 1 & 0.04 \\
\hline Op/Op & 8 & 0.21 & 7 & 0.23 & 3 & 0.24 & 0 & 0.00 & 12 & 0.28 & 2 & 0.07 \\
\hline $\mathrm{Hbl} / \mathrm{Hbl}$ & 19 & 0.50 & 24 & 0.79 & 6 & 0.48 & 12 & 0.20 & 0 & 0.00 & 0 & 0.00 \\
\hline $\mathrm{Bt} / \mathrm{Bt}$ & 3 & 0.08 & 0 & 0.00 & 0 & 0.00 & 0 & 0.00 & 0 & 0.00 & 0 & 0.00 \\
\hline TOT & 3817 & 100 & 3039 & 100 & 1261 & 100 & 5941 & 100 & 4247 & 100 & 2718 & 100 \\
\hline
\end{tabular}


Table 4 Interface types results from a point counting of 100 grains from very coarse $(1-2 \mathrm{~mm})$, coarse (1-0.5 mm) and medium $(0.5-0.25 \mathrm{~mm})$ grain size fractions from Stromboli sand samples (STR=Stromboli Island; STR-9=crater sand. $\mathrm{V} c=$ very coarse, $\mathrm{C}=$ coarse, $\mathrm{m}=$ medium)

\begin{tabular}{|c|c|c|c|c|c|c|c|c|c|c|c|c|}
\hline \multirow{2}{*}{$\begin{array}{l}\text { Interface } \\
\text { type }\end{array}$} & \multicolumn{2}{|c|}{$S T R-1-V c$} & \multicolumn{2}{|c|}{ STR-1-C } & \multicolumn{2}{|c|}{ STR-1-m } & \multicolumn{2}{|c|}{ STR-9-VC } & \multicolumn{2}{|c|}{ STR-9-C } & \multicolumn{2}{|c|}{ STR-9-m } \\
\hline & $\mathrm{N}$. & $\%$ & $\mathrm{~N}$. & $\%$ & $\mathrm{~N}$. & $\%$ & $\mathrm{~N}$. & $\%$ & $\mathrm{~N}$. & $\%$ & $\mathrm{~N}$. & $\%$ \\
\hline Op/Glgr & 0 & 0.00 & 6 & 0.29 & 0 & 0.00 & 21 & 0.65 & 0 & 0.00 & 10 & 0.70 \\
\hline $\mathrm{Pl} / \mathrm{Glgr}$ & 2451 & 65.39 & 1274 & 61.52 & 756 & 53.88 & 1385 & 43.13 & 926 & 49.81 & 540 & 37.74 \\
\hline Px/Glgr & 441 & 11.77 & 195 & 9.42 & 131 & 9.34 & 325 & 10.12 & 187 & 10.06 & 143 & 9.99 \\
\hline $\mathrm{Ol} / \mathrm{Glgr}$ & 299 & 7.98 & 190 & 9.17 & 203 & 14.47 & 332 & 10.34 & 240 & 12.91 & 269 & 18.80 \\
\hline $\mathrm{Hbl} / \mathrm{Glgr}$ & 3 & 0.08 & 0 & 0.00 & 7 & 0.50 & 18 & 0.56 & 3 & 0.16 & 8 & 0.56 \\
\hline Idd/Glgr & 0 & 0.00 & 0 & 0.00 & 0 & 0.00 & 8 & 0.25 & 3 & 0.16 & 4 & 0.28 \\
\hline $\mathrm{Bt} / \mathrm{Glgr}$ & 0 & 0.00 & 0 & 0.00 & 2 & 0.14 & 0 & 0.00 & 0 & 0.00 & 0 & 0.00 \\
\hline $\mathrm{Rt} / \mathrm{Glgr}$ & 0 & 0.00 & 0 & 0.00 & 0 & 0.00 & 0 & 0.00 & 0 & 0.00 & 0 & 0.00 \\
\hline $\mathrm{Pl} / \mathrm{Migr}$ & 0 & 0.00 & 0 & 0.00 & 0 & 0.00 & 0 & 0.00 & 0 & 0.00 & 0 & 0.00 \\
\hline Px/Migr & 0 & 0.00 & 0 & 0.00 & 0 & 0.00 & 0 & 0.00 & 0 & 0.00 & 0 & 0.00 \\
\hline Op/Migr & 0 & 0.00 & 0 & 0.00 & 0 & 0.00 & 0 & 0.00 & 0 & 0.00 & 0 & 0.00 \\
\hline Ol/Migr & 0 & 0.00 & 0 & 0.00 & 0 & 0.00 & 0 & 0.00 & 0 & 0.00 & 0 & 0.00 \\
\hline $\mathrm{Hbl} / \mathrm{Migr}$ & 0 & 0.00 & 0 & 0.00 & 0 & 0.00 & 0 & 0.00 & 0.00 & 0.00 & 0 & 0.00 \\
\hline Rt/Migr & 0 & 0.00 & 0 & 0.00 & 0 & 0.00 & 0 & 0.00 & 0 & 0.00 & 0 & 0.00 \\
\hline Bt/Migr & 0 & 0.00 & 0 & 0.00 & 0 & 0.00 & 0 & 0.00 & 0 & 0.00 & 0 & 0.00 \\
\hline Op/OI & 8 & 0.21 & 73 & 3.52 & 81 & 5.77 & 70 & 2.18 & 51 & 2.74 & 81 & 5.66 \\
\hline $\mathrm{Px} / \mathrm{Ol}$ & 31 & 0.83 & 3 & 0.14 & 14 & 1.00 & 202 & 6.29 & 43 & 2.31 & 16 & 1.12 \\
\hline $\mathrm{Pl} / \mathrm{Ol}$ & 11 & 0.29 & 13 & 0.63 & 19 & 1.35 & 116 & 3.61 & 30 & 1.61 & 18 & 1.26 \\
\hline $\mathrm{PI} / \mathrm{Op}$ & 12 & 0.32 & 3 & 0.14 & 0 & 0.00 & 6 & 0.19 & 36 & 1.94 & 56 & 3.91 \\
\hline Px/Op & 128 & 3.42 & 119 & 5.75 & 29 & 2.07 & 104 & 3.24 & 65 & 3.50 & 123 & 8.60 \\
\hline $\mathrm{Pl} / \mathrm{Px}$ & 100 & 2.67 & 55 & 2.66 & 21 & 1.50 & 135 & 4.20 & 26 & 1.40 & 15 & 1.05 \\
\hline $\mathrm{Ol} / \mathrm{ldd}$ & 0 & 0.00 & 2 & 0.10 & 0 & 0.00 & 19 & 0.59 & 19 & 1.02 & 4 & 0.28 \\
\hline $\mathrm{Hbl} / \mathrm{Pl}$ & 0 & 0.00 & 0 & 0.00 & 0 & 0.00 & 0 & 0.00 & 0 & 0.00 & 0 & 0.00 \\
\hline $\mathrm{Hbl} / \mathrm{Px}$ & 0 & 0.00 & 0 & 0.00 & 0 & 0.00 & 5 & 0.16 & 2 & 0.11 & 4 & 0.28 \\
\hline $\mathrm{Bt} / \mathrm{Pl}$ & 0 & 0.00 & 0 & 0.00 & 0 & 0.00 & 0 & 0.00 & 0 & 0.00 & 0 & 0.00 \\
\hline $\mathrm{Bt} / \mathrm{Px}$ & 0 & 0.00 & 0 & 0.00 & 2 & 0.14 & 0 & 0.00 & 0 & 0.00 & 0 & 0.00 \\
\hline $\mathrm{Bt} / \mathrm{Op}$ & 0 & 0.00 & 0 & 0.00 & 0 & 0.00 & 0 & 0.00 & 0 & 0.00 & 0 & 0.00 \\
\hline $\mathrm{Hbl} / \mathrm{Op}$ & 0 & 0.00 & 0 & 0.00 & 0 & 0.00 & 0 & 0.00 & 0 & 0.00 & 8 & 0.56 \\
\hline Idd/Px & 0 & 0.00 & 0 & 0.00 & 0 & 0.00 & 0 & 0.00 & 0 & 0.00 & 0 & 0.00 \\
\hline$|d d / P|$ & 0 & 0.00 & 0 & 0.00 & 0 & 0.00 & 0 & 0.00 & 0 & 0.00 & 0 & 0.00 \\
\hline $\mathrm{Ol} / \mathrm{Bt}$ & 0 & 0.00 & 0 & 0.00 & 0 & 0.00 & 0 & 0.00 & 0 & 0.00 & 0 & 0.00 \\
\hline $\mathrm{Ol} / \mathrm{Hbl}$ & 0 & 0.00 & 0 & 0.00 & 12 & 0.86 & 0 & 0.00 & 0 & 0.00 & 0 & 0.00 \\
\hline $\mathrm{Bt} / \mathrm{Hbl}$ & 0 & 0.00 & 0 & 0.00 & 0 & 0.00 & 0 & 0.00 & 0 & 0.00 & 0 & 0.00 \\
\hline $\mathrm{Pl} / \mathrm{PI}$ & 190 & 5.07 & 114 & 5.50 & 103 & 7.34 & 343 & 10.68 & 192 & 10.33 & 98 & 6.85 \\
\hline $\mathrm{Px} / \mathrm{Px}$ & 47 & 1.25 & 16 & 0.77 & 13 & 0.93 & 75 & 2.34 & 15 & 0.81 & 9 & 0.63 \\
\hline $\mathrm{Ol} / \mathrm{Ol}$ & 27 & 0.72 & 8 & 0.39 & 10 & 0.71 & 47 & 1.46 & 21 & 1.13 & 25 & 1.75 \\
\hline Op/Op & 0 & 0.00 & 0 & 0.00 & 0 & 0.00 & 0 & 0.00 & 0 & 0.00 & 0 & 0.00 \\
\hline $\mathrm{Hbl} / \mathrm{Hbl}$ & 0 & 0.00 & 0 & 0.00 & 0 & 0.00 & 0 & 0.00 & 0 & 0.00 & 0 & 0.00 \\
\hline $\mathrm{Bt} / \mathrm{Bt}$ & 0 & 0.00 & 0 & 0.00 & 0 & 0.00 & 0 & 0.00 & 0 & 0.00 & 0 & 0.00 \\
\hline TOT & 3748 & 100 & 2071 & 100 & 1403 & 100 & 3211 & 100 & 1859 & 100 & 1431 & 100 \\
\hline
\end{tabular}




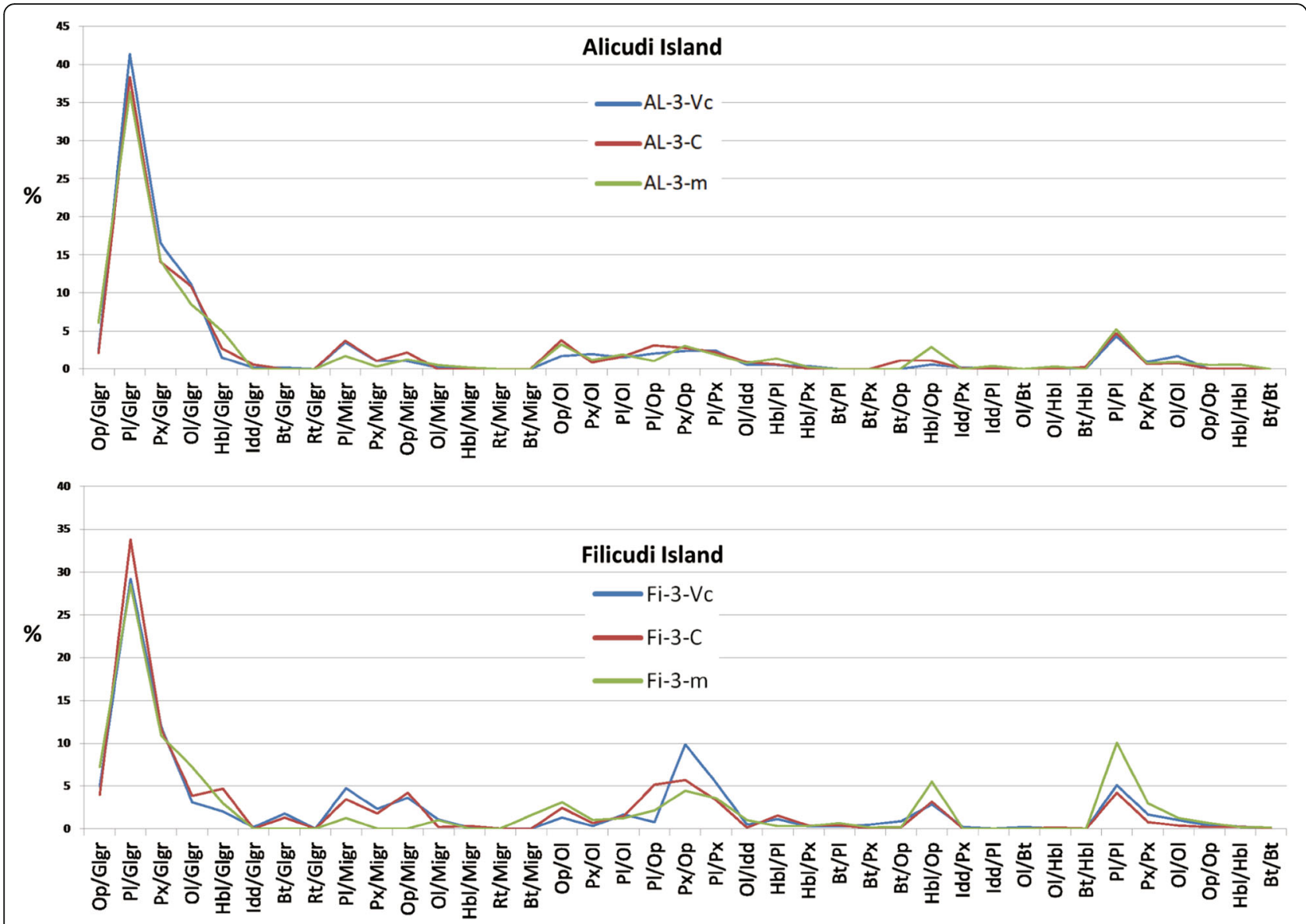

Fig. 3 Interface typologies of counted interfaces percentages of Alicudi and Filicudi islands. $\mathrm{V} c=$ very coarse beach sand, $\mathrm{C}=\mathrm{coarse}$ beach sand, $\mathrm{m}=$ medium beach sand

cumulatively a loss of $50 \%$ is registered from very coarse to medium sand for first-cycle volcaniclastic beach sand (Fig. 6h). Pl/Glgr and Px/Glgr are glass-rich noisostructural interfaces (Table 1, Fig. 6h) and in Filicudi Island beach sand both decrease with a similar disintegration rate: $\mathrm{Pl} / \mathrm{Glgr}$ interface decrease of $38 \%$ and $\mathrm{Px} / \mathrm{Glgr}$ interface loss their $40 \%$, from very coarse to medium sand (Fig. 6h). The isomineralic $\mathrm{Pl} / \mathrm{Pl}$ (Plagioclase/Plagioclase interface; Table 1) shows a decrease during the transition from very coarse to coarse sand $(-19 \%)$ and nearly equal to $3 \%$ during the conversion from coarse to medium with a cumulative loss of $21 \%$ from very coarse to medium sand (Fig. $6 \mathrm{~h}$ ). These data suggest that the iso-structural $\mathrm{Pl} / \mathrm{Pl}$ linear boundary (e.g., Riber et al. 2016, 2017), constituted by interlocked plagioclase crystals, is more stable than both glass-rich interfaces (Pl/Glgr and $\mathrm{Px} / \mathrm{Glgr}$ ) and of the noisomineralic interfaces $(\mathrm{Px} / \mathrm{Op})$. However, a decrease of $21 \%$ in the percentage of $\mathrm{Pl} / \mathrm{Pl}$ interfaces can be related to the active reworking of sandy detritus in the beach environment of the Alicudi Island likewise fluvial (e.g., Heins 1995) and glacial environments (e.g., Caracciolo et al. 2012) that can disrupt and weaken a polycrystalline grain enhancing intercrystals fracturing (e.g., Slatt and Eyles 1981).

\subsection{Salina Island (SA-1)}

Interface types range between 20 and 24 categories with a total of 5941 in the very coarse sand, 4247 in the coarse sand and 2718 in the medium sand counted categories, respectively (Table 3). Pl/Glgr (Plagioclase/Glassy groundmass), Px/Glgr (Pyroxene/ Glassy groundmass) and Px/Migr (Pyroxene/Microlitic groundmass) are the three interfacial heterogeneous types dominating among other less abundant interfacial types (Table 3 and Fig. 4). The conversion from very coarse to coarse sand led to a percentage decrease, in all of the interfaces types, equal to $13.12 \%$ and to $11.85 \%$ from the coarse to the medium grain size, respectively (Fig. 6c). Cumulatively, the conversion from very coarse to medium sand grain size is related to a decrease of $25.24 \%$ of the overall interfaces types. 


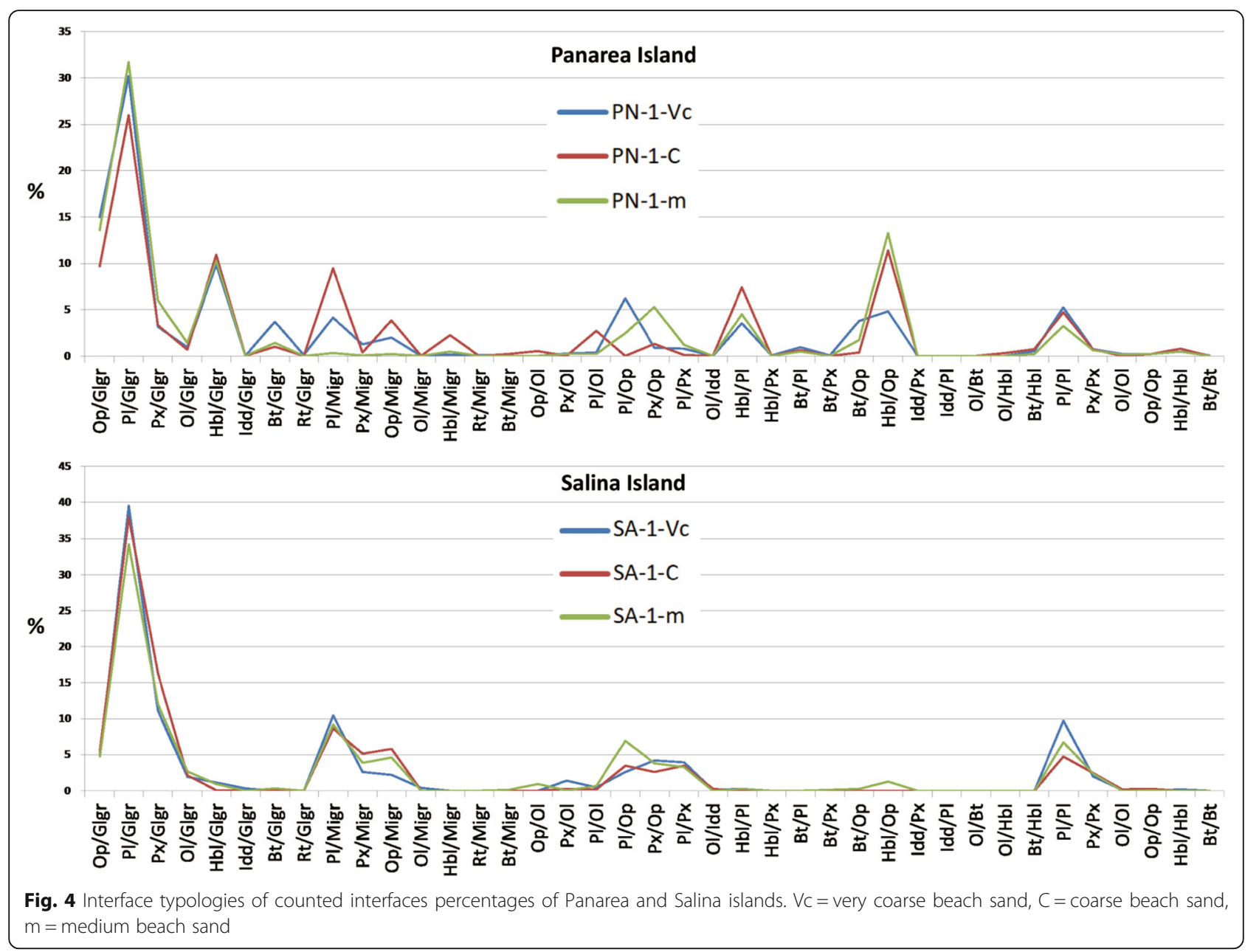

\subsection{Panarea Island (PN-1)}

Interface types range between 25 and 30 categories with a total of 3817 in the very coarse sand, 3039 in the coarse sand and 1261 in the medium sand counted categories, respectively (Table 3). Pl/Glgr (Plagioclase/Glassy groundmass), Op/Glgr (Opaque/Glassy groundmass) and $\mathrm{Hbl} /$ Glgr (Hornblende/Glassy groundmass) are the three interfacial no-isostructural types dominating among other less abundant interfacial types for the very coarse sand; $\mathrm{Pl} /$ Glgr, $\mathrm{Hbl} / \mathrm{Op}$ (Hornblende/Opaque) and $\mathrm{Hbl} / \mathrm{Glgr}$ for the coarse sand and, $\mathrm{Pl} / \mathrm{Glgr}, \mathrm{Op} / \mathrm{Glgr}$ and $\mathrm{Hbl} / \mathrm{Op}$ for the medium sand (Table 3, Fig. 4). The conversion from very coarse to coarse sand led to a percentage decrease equal to $9.58 \%$, and to $21.9 \%$ from the coarse to the medium grain size, respectively, for all of the interface types (Fig. 6d). Cumulatively, the conversion from very coarse to medium sand grain size is related to a decrease of $31.48 \%$ of the overall interface types.

\subsection{Stromboli Island (STR-1)}

Interface types range between 13 and 15 categories with a total of 3748 in the very coarse sand, 2071 in the coarse sand and 1403 in the medium sand counted categories, respectively (Table 4). The interfacial composition of the very coarse, coarse and medium sand is identical: $\mathrm{Pl} / \mathrm{Glgr}$ (Plagioclase/Glassy groundmass), $\mathrm{Px} /$ Glgr (Pyroxene/Glassy groundmass) and Ol/Glgr (Olivine/ Glassy groundmass) are the three interfacial heterogeneous types dominating among other less abundant interfacial types (Table 4, Fig. 5). As in the other islands beach sand, compositional variation, within the sand of Stromboli beach, is accompanied by interfacial percentage decreasing with decreasing grain size: the conversion from very coarse to coarse sand led to a percentage decrease, in all of the interface types, equal to $23.22 \%$ and to $9.25 \%$ from the coarse to the medium grain size, respectively (Fig. 6e). Cumulatively, the conversion from very coarse to medium sand grain size is related to a decrease of $32.47 \%$ of the whole interface types (Fig. 6e).

\subsection{Stromboli Island (STR-9)}

The sampled crater sand is produced in a setting relating to its specific source rock where the potential mechanical loss of sand interfaces is controlled by aeolian 


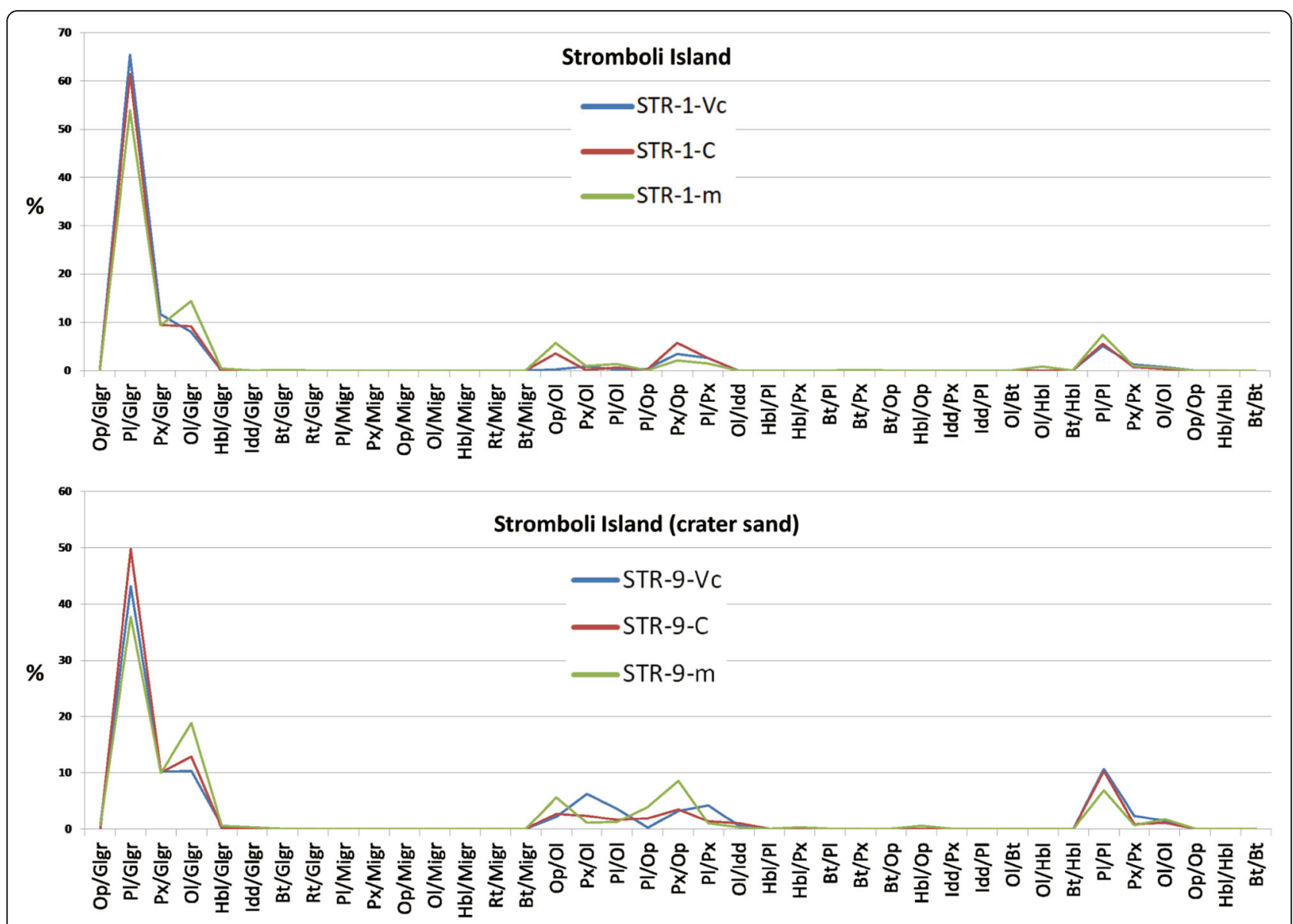

Fig. 5 Interface typologies of counted interfaces percentages of Stromboli beach (upper) and crater (lower) sand. Vc=very coarse beach sand, $C=$ coarse beach sand,$m=$ medium beach sand

transport instead of fluvial or coastal transport mechanisms (e.g., Ingersoll 1990; Ingersoll et al. 1993). Interface types range between 16 and 18 categories with a total of 3211 in the very coarse sand, 1859 in the coarse sand and 1431 in the medium sand counted categories, respectively (Table 4). The three interfacial heterogeneous types, dominating among other less abundant interfacial types, are $\mathrm{Pl} / \mathrm{Glgr}, \mathrm{Pl} / \mathrm{Pl}$ and $\mathrm{Ol} / \mathrm{Glgr}$ for the very coarse and coarse sand, and $\mathrm{Pl} / \mathrm{Glgr}, \mathrm{Ol} / \mathrm{Glgr}$ and $\mathrm{Px} / \mathrm{Glgr}$ for the medium sand (Table 4, Fig. 5). Compositional variation, within the sand of Stromboli crater, related to wind flow dynamics, is accompanied by interfacial percentage decreasing with decreasing grain size analogue to those controlled by reworking at beach environment. In fact, the conversion from very coarse to coarse sand led to a percentage decrease, in all of the interface types, equal to $20.79 \%$ and to $6.59 \%$ from the coarse to the medium grain size, respectively (Fig. 6f). Cumulatively, the conversion from very coarse to medium sand grain size is related to a decrease of $27.38 \%$ of the overall interface types.

\section{Discussion}

Sand-sized grains may be produced by five basic processes that are 1) weathering, both disintegration and chemical decomposition; 2) explosive volcanism (pyroclastic); 3) crushing, both by rock movements (cataclastic) and impact; 4) pelletization, and 5) precipitation from solution, both chemical and biochemical (e.g., Pettijohn et al. 1987). Those grains relating to weathering have been termed epiclastic, and, according to Garrels and MacKenzie (1971), volcanogenic rocks form $26 \%$ of the total sedimentary mass and, of this, a significant part is volcanic sand. In the Aeolian islands, chemical weathering and leaching potential operate at low rate (Garzanti and Andò 2007a, 2007b) but, conversely, steep gradients in rocks and soils, sparse vegetation cover and strong winds, are favorable to a physical weathering of the source rocks producing epiclastic sand mainly in the range of very coarse, coarse and medium grain-sizes (e.g., Critelli et al. 1993; Morrone et al. 2017, 2018) with a detrital mineral composition strongly sizedependent for both light (Morrone et al. 2017) and 

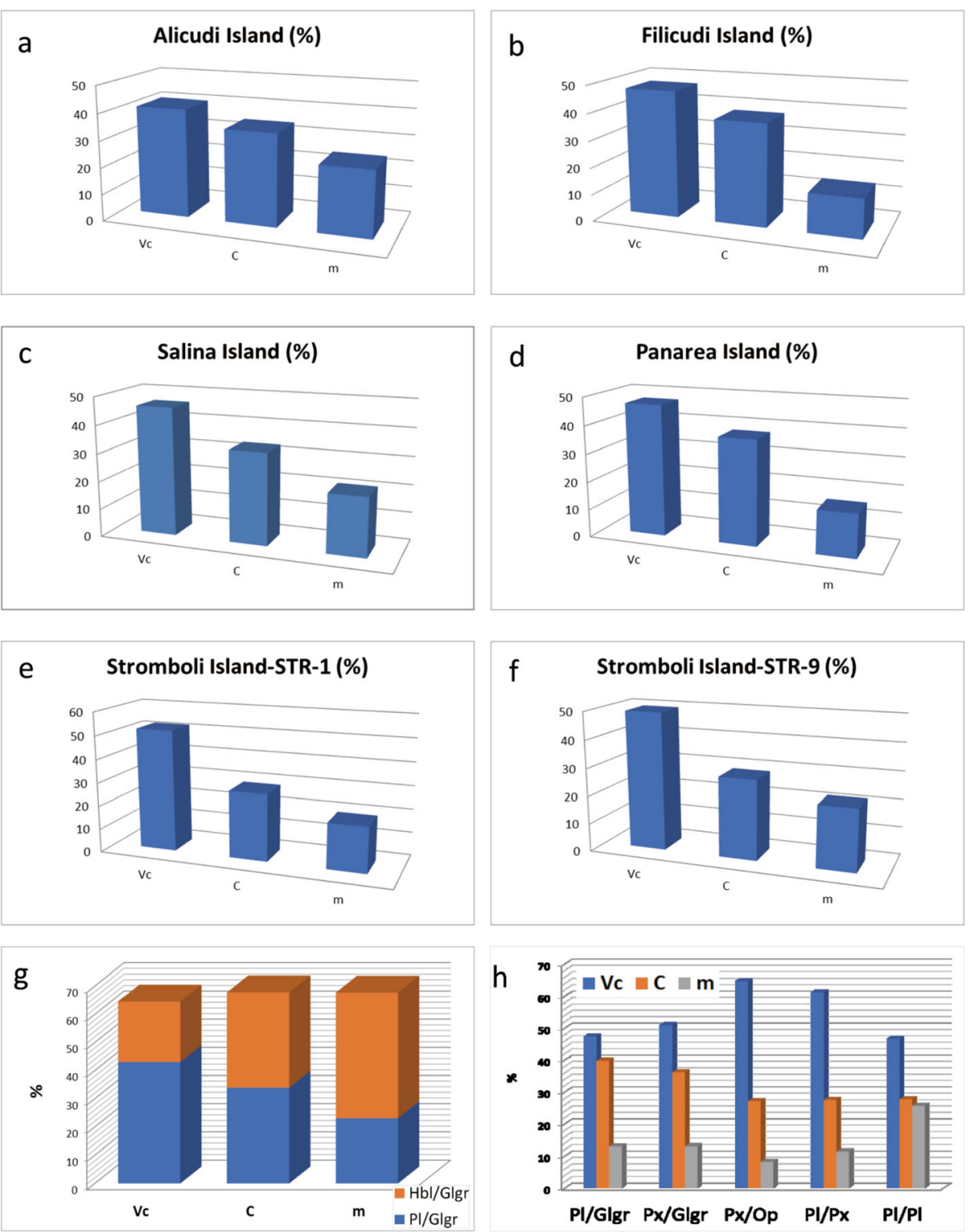

Fig. $\mathbf{6}$ Interfacial percentage decreasing with decreasing grain size of the analyzed beach sand samples (a-f). $\mathrm{V} c=$ very coarse sand; $\mathrm{C}=\mathrm{coarse}$ sand; $\mathrm{m}=$ medium sand. $\mathbf{g}$-Decreasing (Pl/Glgr) or increasing ( $\mathrm{Hbl} / \mathrm{Glgr}$ ) abundance of some glass-rich no-isostructural interfaces of Alicudi very coarse (Vc), coarse (C) and medium (m) sand; h-Relationship between no-isomineralic (Pl/Glgr; Px/Glgr; Px/Op; Pl/Px) and isomineralic interfaces (PI/PI) of Filicudi Island beach sands

heavy minerals (Garzanti and Andò 2007a) within the sandy fraction.

\subsection{No-isostructural interfaces types}

The modal mineralogy analysis (Tables 2,3 and 4) indicates that lathwork and porphyritic volcanic lithic grains of the five Aeolian archipelago beaches, and in those of one crateric sand (STR-9 sample), are dominated by the no-isostructural $\mathrm{Pl} / \mathrm{Glgr}$ interface. This boundary, between plagioclase and the glassy groundmass (Pl/Glgr), occurs as dominant interfacial composition in all of the three sand subfractions of the Aeolian island beaches volcanic lithic grains, as well as in those of the crateric sand of Stromboli volcano. Second as in abundance, noisostructural interface type is represented by pyroxene phenocryst against a glassy groundmass (Px/Glgr interface type of Table 1). Px/Glgr interface occurs in the three sand subfractions of Alicudi, Filicudi, Salina and Stromboli islands whereas it lacks within the sand of Panarea Island beach. As regards Stromboli crateric centre sand, the higher percentage of the Px/Glgr interface occurs in the medium sand fraction. By contrast, this interface type is absent from Panarea Island volcanic lithic grains where opaque (Op/Glgr) and hornblende 
(Hbl/Glgr) phenocrystals commonly define the boundary with the glassy groundmass. Pyroxene phenocrysts, other than with a glassy groundmass, have been found as having a boundary with microlitic groundmass (Px/Migr), or with an opaque mineral (Px/Op), in the three sand subfractions of the lithic grains of Salina and Filicudi island beaches. As regard the interface between olivine, as phenocryst, and the glassy groundmass $(\mathrm{Ol} / \mathrm{Glgr})$, this is concentrated in all the subfractions of Stromboli Island sand, both beach and crateric centre, and in those of Alicudi beach. The Panarea Island lithic grains of the sampled beach sand contain different types and proportions of no-isostructural interfaces: the $\mathrm{Pl} / \mathrm{Glgr}$ interface is concentrated in the three subfractions as in the other islands sand. In addition to plagioclase $(\mathrm{Pl})$ phenocrystal, also opaque $(\mathrm{O})$ and hornblende $(\mathrm{Hbl})$ phenocrystals, yield interfaces with a glassy groundmass (Glgr), in all the three sand subfractions. Interfaces involving opaques (O) occur along boundaries with hornblende ( $\mathrm{Hbl} / \mathrm{Op}$ interface) in the coarse and medium sand.

Volcanic lithic grains of the Aeolian islands sand are characterized by glass-rich interfaces and the dominant nature of the source rocks, including phenocrysts-rich basaltic, basaltic andesitic and andesite lava flows and pyroclastic rocks, and minor dacites, latites and rhyolites (Lucchi 2013), have certainly an influence on the compositions, textures and abundance of these interface types. However, in addition to the source lithotypes controls on sand composition (e.g., Heins and Kairo 2007), abrasion and breakdown of the grains, during the transit from ephemeral stream channels to beach environment (e.g., Mack 1978; Le Pera and Critelli 1997; Morrone et al. 2017, 2018), should be considered to understand the mechanical durability of these no-isostructural interfaces, and which of them are preferentially retained or removed at beach environment.

The no-isomineralic interfaces control mineral disaggregation of the eroded source rocks and, for plutoniclastic sand, during grain-size reduction by comminution, labile interfaces connecting a phyllosilicate (for instance biotite), or an opaque, with a tectosilicate (for instance quartz), are the most sensitive interfaces to the environmental controls whereas the most chemically durable interfaces are $\mathrm{QQ}>>\mathrm{KK}>>\mathrm{PlPl}$, in this order (e.g., Heins 1995).

Comminution of glass-rich volcanic lithic sandy grains, along interfaces, has not been quantified before as a factor leading to the mechanical disruption of composite grains to monocrystalline ones. Davies et al. (1978) suggest that a volcanic rock fragment, with phenocrysts in an aphanitic groundmass, quickly disintegrate by impact shattering in high-gradient streams into glass, grains composed of small crystals and glass, and large crystals with thin glass crust, emphasizing the role of impact shattering of sand grains to explain the observed downstream destruction of rock fragments and the liberation of free (mono)crystals. Cather and Folk (1991), in their study on andesitic detritus, in a semiarid climate, concluded that fractionation of phenocrystals from groundmass dominantly occurs due to both abrasion and impact shattering during transport. Groundmass, and devitrified products, would result more readily comminuted to silt size whereas phenocryst phases, would be overrepresented in sand sizes fractions of the volcaniclastic detritus. Moreover, these authors state that impact shattering may cause effective mineralogic disaggregation of smaller fragments like sand and silt as the length of intercrystalline boundaries approaches the diameter of these smaller grains. In a previous study on volcaniclastic sand on the Aeolian archipelago (Morrone et al. 2018), the quantitative roundness analysis for the Lipari Island beach sand, indicates that mechanical preservation potential for this sedimentary environment is $\mathrm{Lvv}>\mathrm{Lvf}>$ Lvl $>$ Lvmi [(volcanic lithic grains with vitric (Lvv) $>$ felsitic (Lvf) > lathwork (Lvl) > microlitic (Lvmi) textures], for volcanic lithic grains of the medium sand fraction. The comminution of more brittle vitric (Lvv) lithic grains may result in grain fracture, splintering them into fragment, whereas felsitic (Lvf), lathwork (Lvl) and microlitic (Lvmi) volcanic particles, are primarily influenced by rounding through abrasion, with roundness categories ranging from 3 (sub-rounded grains) to 6 (well rounded grains). As regards, the sand fractions of the present study, despite we have not estimated quantitatively the roundness, as Morrone et al. (2018) did for Lipari beach sand, sandy grains with no-isostructural interfaces, such as $\mathrm{Pl} / \mathrm{Glgr}, \mathrm{Px} / \mathrm{Glgr}$ and $\mathrm{Ol} / \mathrm{Glgr}$, are better rounded than sandy grains containing iso-mineralic interfaces such as $\mathrm{Pl} / \mathrm{Pl}$ or $\mathrm{Px} / \mathrm{Px}$ (Figs. 7, 8, 9 and 10). By contrast, these latter interface types are characterized by more angular shapes of their clasts (Figs. 7, 8, 9 and 10). These observations imply interface types control comminution of volcanic lithic grains into smaller pieces: comminution is chiefly accomplished by abrasion of the glass-rich grains containing noisostructural interfaces, with semi-rounded and rounded grains morphologies, and by breakage along isomineralic boundaries producing mainly volumes of subangular and angular sandy grains.

\subsection{No-isomineralic interfaces types}

Mixed no-isomineralic interfaces, such as those containing biotite, are the least mechanically and chemically durable and highly vulnerable to water that can immediately break the interfaces as soon as these enter the weathering environment (e.g., Heins 1995). Moreover, during weathering, biotite can experience widening of the crystal lattice along cleavage regardless of straight or sinuous cleavage planes geometry (e.g., Le Pera and 

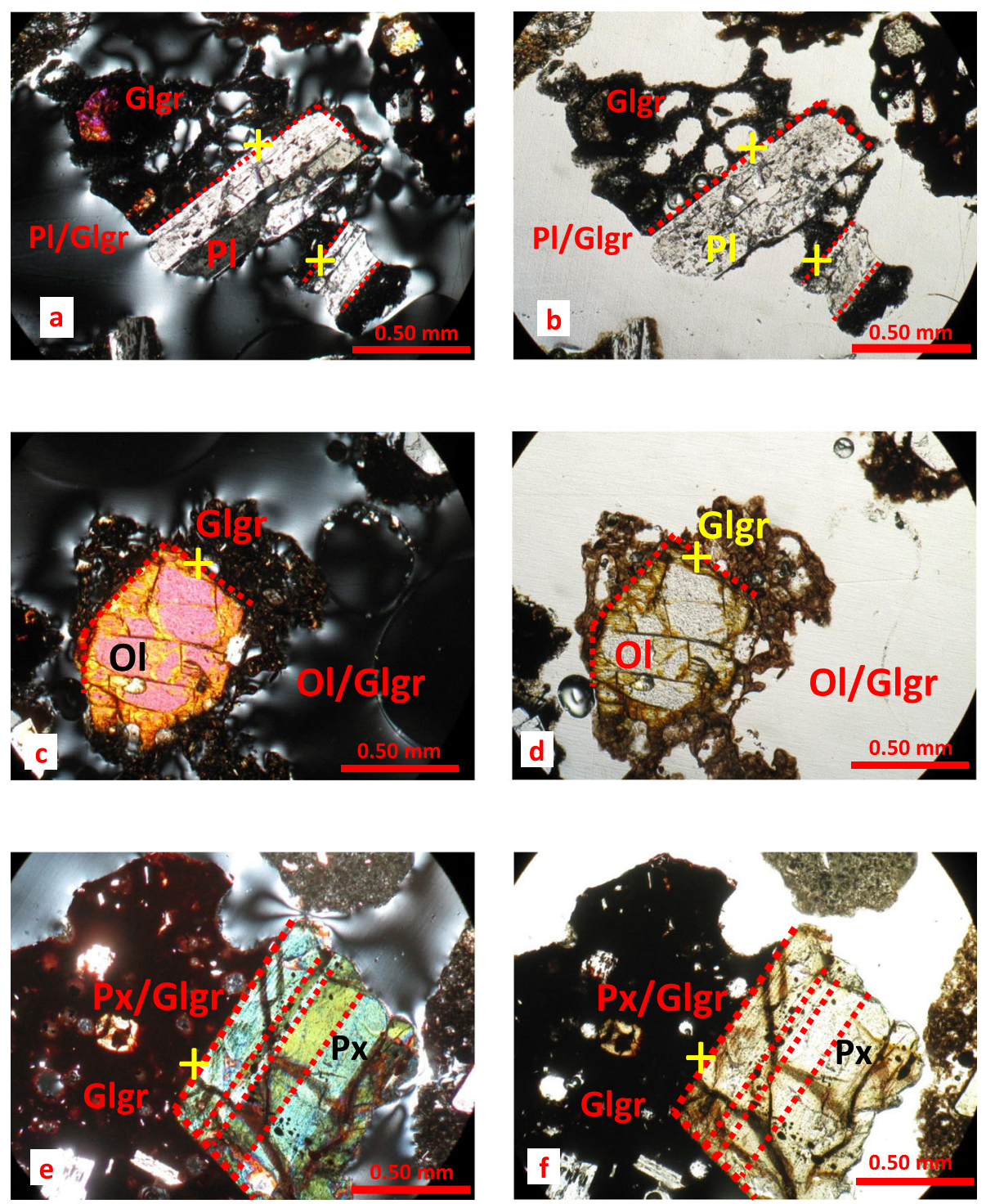

Fig. 7 No-isostructural glass-rich interface between a phenocryst and a glassy groundmass. a, b: Pl/Glgr interface (Plagioclase/Glassy groundmass). a Crossed nicols; b Plane-polarized light; c, d: Ol/Glgr interface (Olivine/Glassy groundmass); c Crossed nicols; d Plane-polarized light; e, f: Px/Glgr interface (Pyroxene/Glassy groundmass); e Crossed nicols; $\mathbf{f}$ Plane-polarized light. Twin domains of plagioclase (a, b), olivine fracture (c, d) and pyroxene cleavage $(\mathbf{e}, \mathbf{f})$ are intracrystals interfacial features [10x, Very coarse sand $(2.00-1.00 \mathrm{~mm}) ;+=$ polarizing microscope crosshair]

Sorriso-Valvo 2000; Scarciglia et al. 2012; Riber et al. 2016, 2017). Biotite, in the Aeolian islands sandy grains, can be adjacent to an opaque, a plagioclase, a pyroxene or to an hornblende crystal, defining mixed noisomineralic interfaces such as Phyllosilicate/Opaque, Phyllosilicate/Tectosilicate, or Phyllosilicate/Inosilicate, respectively. These interface types occur in trace amounts in the three subfractions of Alicudi, Filicudi and Salina beach sands and only in the medium sand of Stromboli beach. In the crateric sand of Stromboli some traces of $\mathrm{Bt} / \mathrm{Glgr}$ and of $\mathrm{Bt} / \mathrm{Px}$ occur only in the medium sand. The highest percentage of $\mathrm{Bt} / \mathrm{Op}$ and $\mathrm{Bt} / \mathrm{Glgr}$ interfaces occur in the three subfractions of Panarea
Island sand beach. Rarely, except than in the very coarse sand of Panarea Island beach, it forms a noisostructural interface with a glassy or a microlitic groundmass (Table 3).

Interfaces pertaining to opaque crystals are more complex than those involving biotite (Tables 2, 3 and 4). Opaque phenocrystals are considered as a unique category of mineral forming an interface with another one mineral category, or with a groundmass, rather than subdivided, because reflected light microscopy is not sufficient to discriminate among magnetite, ilmenite, pyrite and hematite, and among their intergrown phases and textures (e.g. Basu and Molinaroli 1989, 1991; 

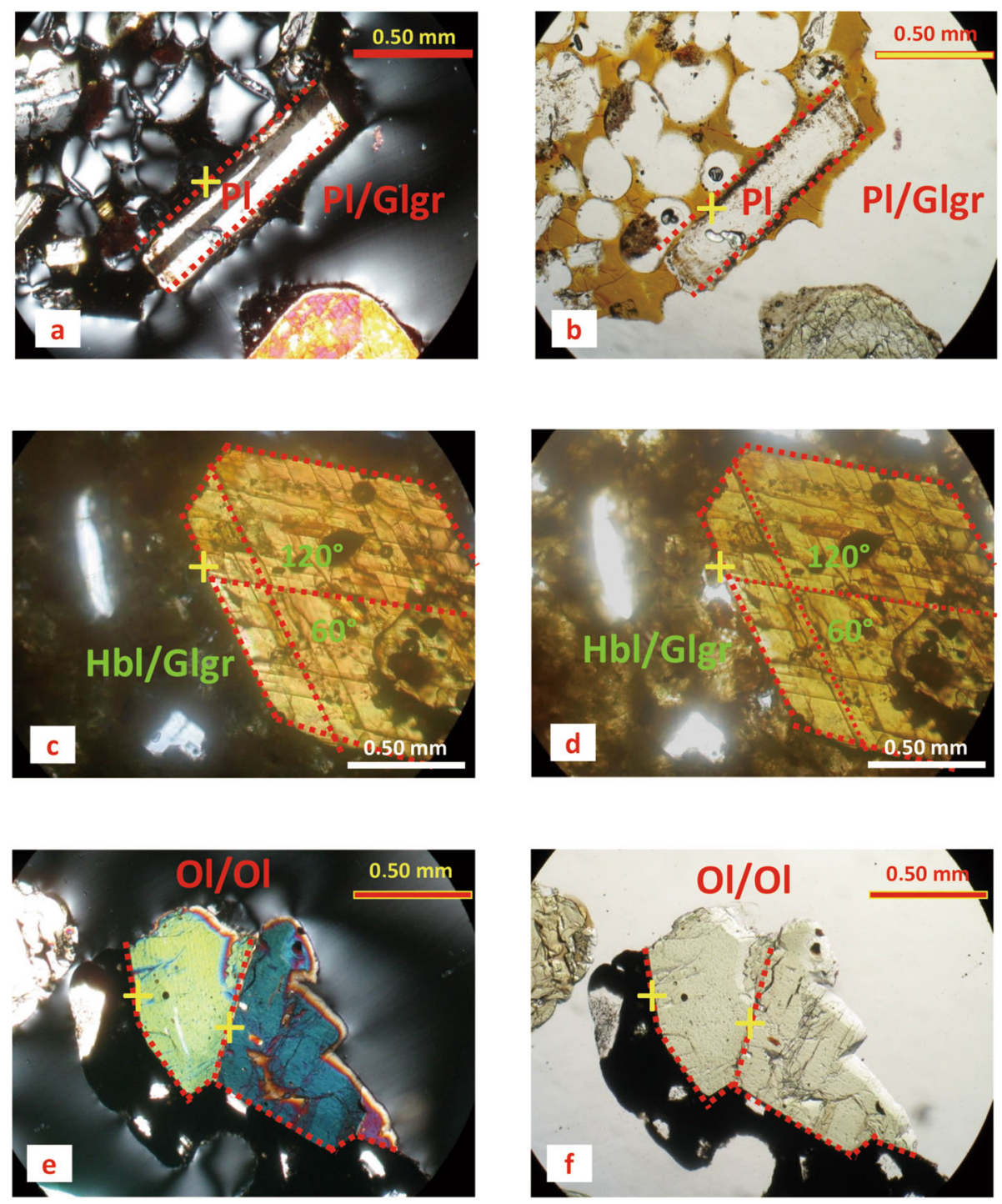

Fig. 8 No-isostructural glass-rich interface between a phenocryst and a glassy groundmass (a-f) and isomineralic (e-f). $\mathbf{a}, \mathbf{b}$ : Pl/Glgr interface (Plagioclase/Vesiculated glassy groundmass. a Crossed nicols: b Plane-polarized light: c, d: Hbl/Glgr interface (Hornblende/Glassy groundmass): c Crossed nicols: d Plane-polarized light: e, f: Ol/OI (Olivine/Olivine interface) and Ol/Glgr (Olivine/Glassy groundmass) interfaces. e Crossed nicols, f Plane-polarized light. The hornblende basal cleavage system $(c, d)$ constitute other preferential weakness planes of intracrystal breakage sites [10x, very coarse sand $(2.00-1.00 \mathrm{~mm})]$

Molinaroli and Basu 1993). Microprobe analysis (e.g., Le Pera and Morrone 2018) or Raman spectroscopy (e.g., Andò and Garzanti 2013) techniques, aimed at geochemical discrimination of opaque detrital minerals, could be used but these are beyond the scope of this paper. Furthermore, in studies concerning plutoniclastic sand, all interface data pertaining to opaque crystals, such as Op/Op (Opaque/Opaque interface), have been left out owing to such uncertainty (e.g., Weltje et al. 2018). In this study the opaques group of countable interfaces include $\mathrm{Op} / \mathrm{Ol}, \mathrm{Op} / \mathrm{Pl}, \mathrm{Op} / \mathrm{Px}, \mathrm{Op} / \mathrm{Hbl}$ and $\mathrm{Op} /$ Bt already discussed, and likewise the other mineral phases, their contact with a glassy or with a microlitic groundmass has been counted, too. Very few grains contains also controversial $\mathrm{Op} / \mathrm{Op}$ interface found as trace amount in four islands beach sand except than those of Stromboli. Filicudi Island beach sand has $\mathrm{Op} /$ Px interface with the highest percentage in all of the three sand subfractions, followed in order of decreasing abundance by Salina, Stromboli, Panarea and Alicudi island beaches, and always showing a drastic decrease with decreasing grain size. $\mathrm{Op} / \mathrm{Hbl}$ interface proportions change among island beaches, with the highest percentage in the Panarea Island sand and the lowest in Stromboli where it has been found as trace amounts only in the medium sand samples at crater. $\mathrm{Op} / \mathrm{Pl}$ is the third 

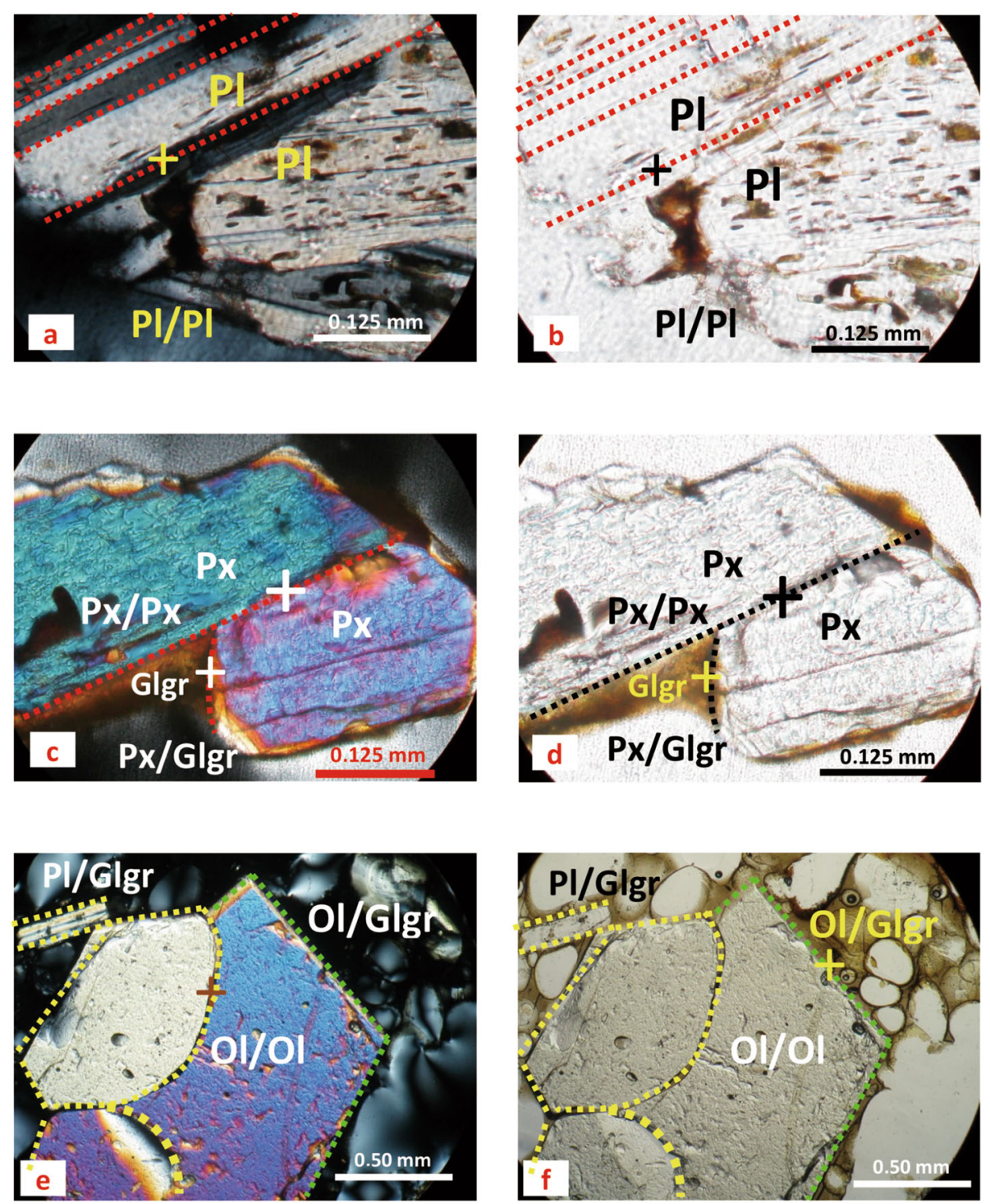

Fig. 9 Iso-mineralic straight $(\mathbf{a}, \mathbf{b})$ and sinuous interfaces $(\mathbf{e}, \mathbf{f})$ and no-isomineralic sinuous and straight (e-f). a, b: PI/PI interface (Plagioclase/ Plagioclase, 50x). a Crossed nicols; b Plane-polarized light; $\mathbf{c}$, d: Ol/OI interface (Olivine/Olivine; 50x). c Crossed nicols; d Plane-polarized light. e, f: $\mathrm{Ol} / \mathrm{Glgr}+\mathrm{Ol} / \mathrm{Ol}+\mathrm{Ol} / \mathrm{Glgr}+\mathrm{Pl} / \mathrm{Glgr}$ interfaces (Olivine/Glassy groundmass + Olivine/Olivine + Olivine/Glassy groundmass + Plagioclase/Glassy groundmass; 10x). e Crossed nicols; f Plane-polarized light. All grains are coarse sand-sized (1.00-0.500 mm)

interface in order of decreasing abundance, with the highest percentage in Panarea Island sand, followed by Filicudi, Salina, Alicudi and Stromboli islands sand. Op/ $\mathrm{Ol}$ is a no-isomineralic interface, with very low mechanical and chemical durabilities (e.g., Heins 1995), and it is more abundant in the Stromboli Island in both crater and beach sand samples, than in the other islands sand (Tables 2, 3 and 4). The highest percentage is contained in the medium sand grain-size category of Stromboli crater sand. During the transition from summit crater sand to beach environment, a sharp drop in $\mathrm{Op} / \mathrm{Ol}$ interface percentage is particularly noticeable in the very coarse sand, with a decrease of at least $62 \%$, whereas it remains almost unchanged in the coarse and medium subfractions (Table 4). Panarea Island beach sand has the highest percentage of interfaces involving opaques (Table 3 ) both as noisomineralic and as no-isostructural interfaces in a contact with a glassy or a microlitic groundmass. Op/Glgr are more abundant than Op/Migr in Panarea, Alicudi, Filicudi and Salina beach sands. In Stromboli Island beach sand $\mathrm{Op} / \mathrm{Glgr}$ interface is present in trace amounts only in the coarse sand subfraction and it lacks Op/Migr interface; the crateric sand has an higher percentage of Op/Glgr with respect to the beach sand implying a loss of this interface type during the transport from the summit crater of Stromboli Island to the conterminous beach environment. 

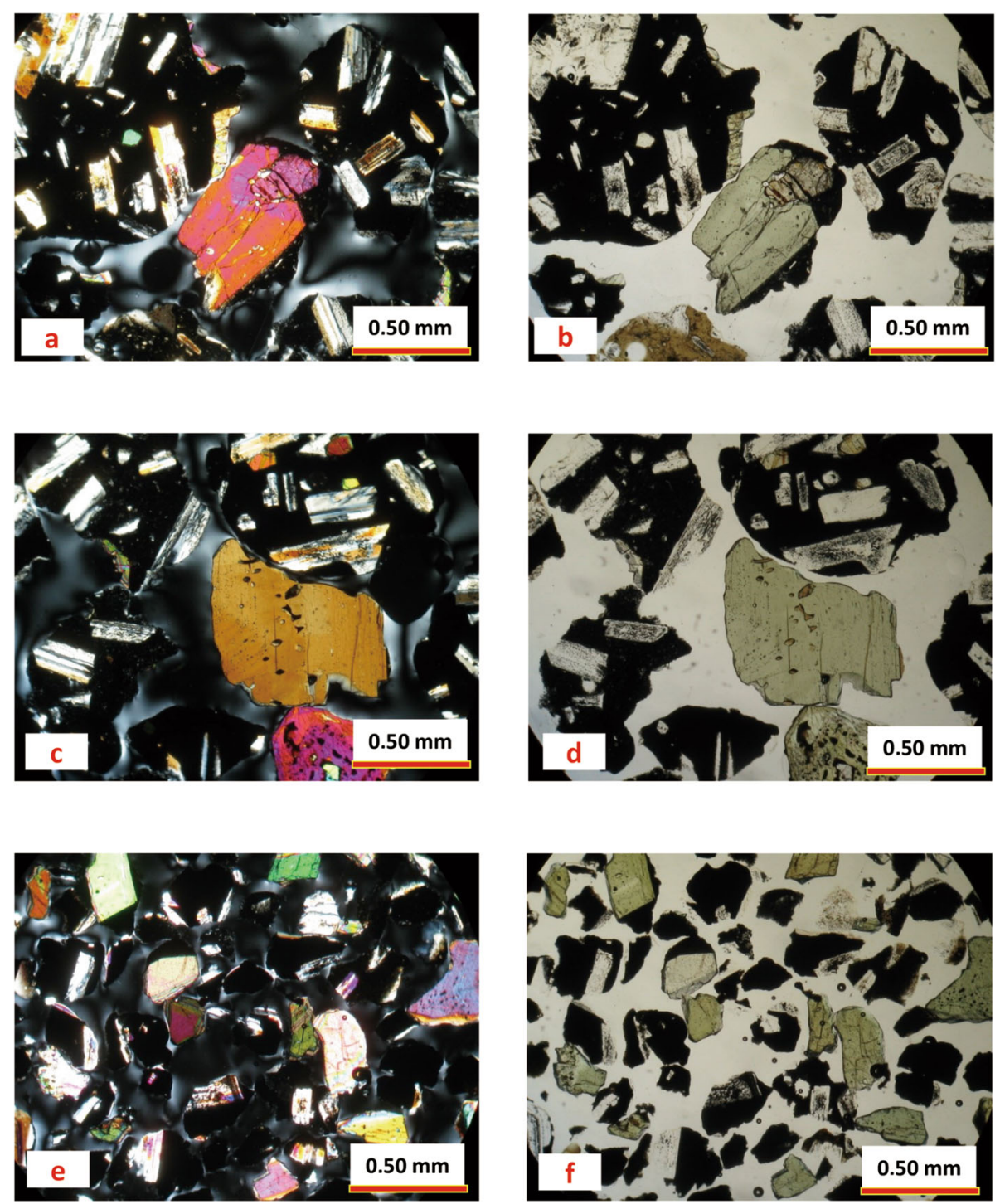

Fig. 10 Relationship between grain size and composition of the sand fraction in Aeolian beaches sand. a, b: Very coarse sand (2.00-1.00 mm; $5 x)$ with only predominant polymineralic volcanic lithic fragments. a Crossed nicols, b Plane-polarized light. c, d: Coarse sand (1.00-0.50 mm) with predominant polymineralic volcanic lithic fragments and few monocrystalline grains ( $1.00-0.500 \mathrm{~mm} ; 5 \mathrm{x})$. c Crossed nicols, d Plane-polarized light. e, f: Medium sand $(0.500-0.250 \mathrm{~mm})$ with predominant monocrystalline grains and minor polymineralic lithic volcanic grains; (5x). e Crossed nicols, f Plane-polarized light

\section{$\mathbf{5 . 3}$ Isomineralic interfaces types}

Counted isomineralic interfaces of the sampled sand are six and their relative mechanical durability can be synthesized as follows, from the most to the less durable category: $\mathrm{Pl} / \mathrm{Pl}>>\mathrm{Px} / \mathrm{Px}>>\mathrm{Ol} / \mathrm{Ol}>>\mathrm{Op} / \mathrm{Op}>>$ $\mathrm{Hbl} / \mathrm{Hbl}>>\mathrm{Bt} / \mathrm{Bt}$. Isostructural tectosilicate contact, such $\mathrm{Pl} / \mathrm{Pl}$ (Plagioclase/Plagioclase) interface, was detected, as the most abundant interface among isomineralic interfaces types (e.g., Heins 1995), in the subfractions of both the five sampled island beaches (Tables 2, 3 and 4) and of the crateric centre of Stromboli volcano sand. Although $\mathrm{Pl} / \mathrm{Pl}$, together with Q/Q (Quartz/Quartz) and K/K (K-feldspar/ $\mathrm{K}$-feldspar) interfaces, is considered one of the interfaces with the highest mechanical preservation potential (e.g., Heins 1995), it constitutes a minor interfacial type of the Aeolian volcaniclastic sand ranking about five or six as in order of abundance interface types (Tables 2, 3 and 4). In this case, transport processes, operating along shortheaded streams with very steep gradients and, later, at a beach environment (e.g., Morrone et al. 2018), could favor a rapid destruction of this isostructural boundary type. This relatively rapid rate of disintegration of the $\mathrm{Pl} / \mathrm{Pl}$ interface, causing lathwork volcanic lithic grains comminution, could be related to the preferential widening of straight mineral boundaries rather than sinuous mineral contacts, a geometric feature prominent for physical 
disintegration of plutoniclastic detritus, too (e.g., Riber et al. 2015). Moreover, stress fields acting on interface categories such as intracrystal and intraplanes of weakness, for instance plagioclase twin planes, have been thought to be the result of plagioclase comminution also in glacial (e.g., Slatt and Eyles 1981; Caracciolo et al. 2012), in deltaic (e.g., Garzanti 1986) environments, or in Arctic climate of Iceland (e.g., Haraldsson 1984). This interface type, lacking in the medium sand fraction of the crateric centre of Stromboli volcano (Table 4), could be disintegrated from ballistic impact, between sand grains, achieved during wind saltation process (e.g., Dutta et al. 1993), at its local scale of outcrop (e.g., Ingersoll 1990; Critelli and Ingersoll 1995), such as the summit crater of Stromboli Island. These detrital grains are particularly susceptible to breakage during transport processes: breakage along their cleavage or twin planes during transport eliminate them from the sandy grain sizes and accumulate in finer grain sizes (e.g., Pittman 1969; Garzanti 1986). In the studied grains, zoning of volcanic plagioclase, impart, between core and rim, a further intracrystalline mechanical anisotropy, relevant to the extent of microcracking during physical weathering (e.g., Heins 1995; Riber et al. 2015; Barros dos Santos et al. 2017; Barros dos Santos et al. 2018). Plagioclase zoning, coupled with the anisotropy proper of the intercrystalline $\mathrm{Pl} / \mathrm{Pl}$ interface, is another control process accelerating the destruction rate of this detrital component. Pyroxene/Pyroxene (Px/Px interface), is second in order of decreasing abundance of isomineralic interfaces except than in the coarse and medium crateric sand of Stromboli that are richer in $\mathrm{Ol} / \mathrm{Ol}$ isomineralic interface (Tables 2, 3 and 4). The ferromagnesian composition and structure of pyroxenes and olivine make them susceptible to faster chemical and physical weathering with respect to other mineral phases (e.g., Eggleton 1986; Velbel 2009; Andò et al. 2012). Most pyroxenes and amphiboles weathering products exhibit preferred crystallographic orientation along fractures and cleavages (e.g, Velbel 1989; Apollaro et al. 2019), also in a weathering-limited erosion regime (e.g., Locke 1986; McCarroll 1990), as well as nanoporosity (e.g., Velbel and Barker 2008), weakening the crystal lattices. So, even if mechanical durability of an isomineralic interface, such as $\mathrm{Px} / \mathrm{Px}$, could be high (e.g., Heins 1995) in the studied sand, the influence of abrasion and mechanical breakdown at beach environment results in a significant reduction of this interface type. Olivine, is the least resistant of the common rock-forming minerals (Goldich 1938; Velbel 1999, 2014) and, in some Ol/Ol counted interfaces, it is weathered to iddingsite along fractures, with the boundary between fresh olivine and iddingsite, promoting intracrystal opening and dislocation along with the Olivine/Olivine interface (Figs. 7, 8, 9 and 10). The Olivine/Olivine interface $(\mathrm{Ol} / \mathrm{Ol})$ is absent in the medium sand of Alicudi Island beach and it occurs as trace amounts in the medium sand fraction of Filicudi, Salina, Panarea and Stromboli beach sand, probably because mechanical abrasion processes on the beach have caused a significant decline in abundance of this interface type. Moreover, despite the Mediterranean climate (ISPRA 2011), which imparts a weathering-limited erosion regime to the Aeolian archipelago source rocks (e.g., Morrone et al. 2017, 2018), other than inorganic processes such iddinsgite rims alteration, some biochemical dissolution processes, due to organic ligands, could have increased the rate of dissolution of olivine (e.g., Grandstaff 1986), promoting further opening of this interface type within source rocks at outcrop scale. Lastly, as regards the boundaries between the $\mathrm{Ol} / \mathrm{Ol}$ domains, both sharp and sinuous interfaces have been observed (Figs. 7, 8, 9 and 10) but not counted separately. Nevertheless, we can expect that sinuous $\mathrm{Ol} / \mathrm{Ol}$ interfaces are more abundant than straight $\mathrm{Ol} /$ $\mathrm{Ol}$ interfaces because, at least as regards plutoniclastic sand, straight crystal interfaces are weaker than more convoluted crystal interfaces which form a tightly interlocked framework (e.g., Weltje et al. 2018).

By considering and comparing the main differences among mechanical preservation potential of the studied sand, general insights emerge when results from the medium sand are analyzed (Table 5).

\section{Conclusions}

This study has been an attempt to understand what would be found in the very coarse, coarse and medium sand fraction in terms of interfaces types, preserved in rock fragments of modern volcaniclastic sand, at a high-energy modern beach environment. The studied sands have a broad range in interfacial compositions, which reflects the complex islands source lithotypes association of volcanic lava flows and pyroclastic deposits. Also, the distribution of some interfaces types, across the grain size intervals, shows a degree of dependence and some possible trends are discernible. The recognized interfaces are 17 of noisomineralic type, 14 of no-isostructural type, and 6 of isomineralic type. Of the 14 no-isostructural types, 8 are interfaces glassy-rich and 6 are glass-poor (or microlitic), as regard the boundary between a phenocrystal and the groundmass. The mechanical preservation potential for the interface type related to the medium sand (e.g., Table 5) has been summarized, in terms of mechanical durabilities, as follows:

\subsection{No-isomineralic interfaces}

\subsubsection{Interfaces involving an opaques crystal and a silicate crystal \\ $\mathrm{Op} / \mathrm{Px}>>\mathrm{Op} / \mathrm{Pl}>>\mathrm{Op} / \mathrm{Hbl}>>\mathrm{Op} / \mathrm{Ol}>>\mathrm{Op} / \mathrm{Bt}$.}

\subsubsection{Interfaces involving an olivine crystal}

$\mathrm{Ol} / \mathrm{Pl}>>\mathrm{Ol} / \mathrm{Px}>>\mathrm{Ol} / \mathrm{Idd}>>\mathrm{Ol} / \mathrm{Hbl}>>\mathrm{Ol} / \mathrm{Bt}(=0)$. 
Table 5 Interface ranking of the mechanical preservation potential

\section{No-isomineralic interfaces}

Interfaces involving an opaque crystal and a silicate crystal: $\mathrm{Op} / \mathrm{Px}>>\mathrm{Op} / \mathrm{Pl}>>\mathrm{Op} / \mathrm{Hbl}>>\mathrm{Op} / \mathrm{Ol}>>\mathrm{Op} / \mathrm{Bt}$

$\begin{array}{ll}\text { Interface } & \text { AL-3-m } \\ \text { Op/Px } & 50 \\ \text { Op/PI } & 17 \\ \text { Op/Hbl } & 48 \\ \text { Op/OI } & 53 \\ \text { Op/Bt } & 0 \\ \text { Interfaces involving } & \text { an olivine crystal: } \\ \text { Ol/PI>> Ol/Px>> OI/Idd > Ol/Hbl } \\ \text { Interface } & \text { AL-3-m } \\ \text { Ol/PI } & 31 \\ \text { Ol/Px } & 19 \\ \text { Ol/Idd } & 13 \\ \text { Ol/Hbl } & 0\end{array}$

$\begin{array}{ll}\text { Fi-3-m } & \text { SA-1-m } \\ 39 & 104 \\ 19 & 189 \\ 48 & 35 \\ 27 & 26 \\ 2 & 8\end{array}$

PN-1-m
67
31
167
0
22

STR-1-m
29
0
0
81
0

$\begin{array}{ll}\text { STR-9-m } & \text { TOT\% } \\ 123 & 30.98 \\ 56 & 23.46 \\ 8 & 23.01 \\ 81 & 20.15 \\ 0 & 2.41\end{array}$

Interfaces involving a phyllosilicate crystal: $\mathrm{Bt} / \mathrm{Op}>>\mathrm{Bt} / \mathrm{Pl}>>\mathrm{Bt} / \mathrm{Px}>>\mathrm{Bt} / \mathrm{Hbl}$

\begin{tabular}{|c|c|c|c|c|c|c|c|}
\hline Interface & AL-3-m & Fi-3-m & SA-1-m & PN-1-m & STR-1-m & STR-9-m & тот\% \\
\hline $\mathrm{Bt} / \mathrm{Op}$ & 0 & 2 & 8 & 22 & 0 & 0 & 58.18 \\
\hline $\mathrm{Bt} / \mathrm{PI}$ & 0 & 6 & 0 & 6 & 0 & 0 & 21.82 \\
\hline $\mathrm{Bt} / \mathrm{Px}$ & 0 & 1 & 5 & 0 & 2 & 0 & 14.55 \\
\hline $\mathrm{Bt} / \mathrm{Hbl}$ & 0 & 0 & 0 & 3 & 0 & 0 & 5.45 \\
\hline \multicolumn{8}{|c|}{$\begin{array}{l}\text { Interfaces involving a pyroxene crystal: } \\
\mathrm{Px} / \mathrm{Pl}>>/ \mathrm{Px} / \text { Horn }>>\mathrm{Px} / \mathrm{Bt}\end{array}$} \\
\hline Interface & AL-3-m & Fi-3-m & SA-1-m & PN-1-m & STR-1-m & STR-9-m & тот\% \\
\hline $\mathrm{Px} / \mathrm{PI}$ & 31 & 31 & 89 & 15 & 21 & 15 & 93.95 \\
\hline $\mathrm{Px} / \mathrm{Hbl}$ & 4 & 3 & 0 & 0 & 0 & 4 & 5.116 \\
\hline $\mathrm{Px} / \mathrm{Bt}$ & 0 & 0 & 0 & 0 & 2 & 0 & 0.93 \\
\hline \multicolumn{8}{|c|}{$\begin{array}{l}\text { Interfaces involving an amphibole crystal: } \\
\mathrm{Hbl} / \mathrm{Op}>>\mathrm{Hbl} / \mathrm{Pl}>>\mathrm{Hbl} / \mathrm{Ol}>>\mathrm{Hbl} / \mathrm{Px}>>\mathrm{Hbl} / \mathrm{Bt}\end{array}$} \\
\hline Interface & AL-3-m & Fi-3-m & SA-1-m & PN-1-m & STR-1-m & STR-9-m & тот\% \\
\hline $\mathrm{Hbl} / \mathrm{Op}$ & 48 & 48 & 35 & 167 & 0 & 8 & 71.50 \\
\hline $\mathrm{Hbl} / \mathrm{PI}$ & 23 & 3 & 8 & 57 & 0 & 0 & 21.26 \\
\hline $\mathrm{Hbl} / \mathrm{Ol}$ & 5 & 0 & 0 & 0 & 12 & 0 & 3.97 \\
\hline $\mathrm{Hbl} / \mathrm{Px}$ & 4 & 3 & 0 & 0 & 0 & 4 & 2.57 \\
\hline $\mathrm{Hbl} / \mathrm{Bt}$ & 0 & 0 & 0 & 3 & 0 & 0 & 0.70 \\
\hline
\end{tabular}

No-isostructural interfaces

Glass-rich:

$\mathrm{Pl} / \mathrm{Glg}>>$ PX/Glgr $>>\mathrm{Ol} / \mathrm{Glg}>>\mathrm{Op} / \mathrm{Glgr}>>\mathrm{Hbl} / \mathrm{Glgr}>>\mathrm{Bt} / \mathrm{Glgr}>>\mathrm{ldd} / \mathrm{Glgr}$

\begin{tabular}{|c|c|c|c|c|c|c|c|}
\hline Interface & AL-3-m & $\mathrm{Fi}-3-\mathrm{m}$ & SA-1-m & PN-1-m & STR-1-m & STR-9-m & тот\% \\
\hline $\mathrm{Pl} / \mathrm{Glgr}$ & 604 & 249 & 929 & 400 & 756 & 540 & 57.42 \\
\hline $\mathrm{Px} / \mathrm{Glgr}$ & 236 & 95 & 328 & 76 & 131 & 143 & 16.66 \\
\hline $\mathrm{Ol} / \mathrm{Glgr}$ & 141 & 63 & 73 & 18 & 203 & 269 & 12.66 \\
\hline Op/Glgr & 101 & 63 & 130 & 171 & 0 & 10 & 7.84 \\
\hline $\mathrm{Hbl} / \mathrm{Glgr}$ & 83 & 26 & 26 & 130 & 7 & 8 & 4.62 \\
\hline $\mathrm{Bt} / \mathrm{Glgr}$ & 0 & 14 & 10 & 18 & 2 & 0 & 0.73 \\
\hline
\end{tabular}


Table 5 Interface ranking of the mechanical preservation potential (Continued)

\begin{tabular}{|c|c|c|c|c|c|c|c|}
\hline Idd/Glgr & 0 & 0 & 0 & 0 & 0 & 4 & 0.07 \\
\hline \multicolumn{8}{|c|}{$\begin{array}{l}\text { Glass-poor or microlitic: } \\
\mathrm{Pl} / \text { Migr }>>\mathrm{Op} / \text { Migr }>>\mathrm{Px} / \text { Migr }>>\mathrm{O} / \text { Migr }>>\mathrm{Hbl} / \text { Migr }\end{array}$} \\
\hline Interface & AL-3-m & Fi-3-m & SA-1-m & PN-1-m & STR-1-m & STR-9-m & тот\% \\
\hline $\mathrm{Pl} /$ Migr & 28 & 11 & 250 & 4 & 0 & 0 & 50.52 \\
\hline Op/Migr & 21 & 0 & 126 & 3 & 0 & 0 & 25.86 \\
\hline Px/Migr & 5 & 0 & 105 & 1 & 0 & 0 & 19.14 \\
\hline Ol/Migr & 8 & 9 & 0 & 0 & 0 & 0 & 2.93 \\
\hline $\mathrm{Hbl} / \mathrm{Migr}$ & 3 & 0 & 0 & 6 & 0 & 0 & 1.55 \\
\hline \multicolumn{8}{|c|}{$\begin{array}{l}\text { Isomineralic interfaces: } \\
\mathrm{Pl} / \mathrm{Pl}>>\mathrm{Px} / \mathrm{Px}>>\mathrm{Ol} / \mathrm{Ol}>>\mathrm{Op} / \mathrm{Op}>>\mathrm{Hb} / / \mathrm{Hbl}>>\mathrm{Bt} / \mathrm{Bt}\end{array}$} \\
\hline Interface & AL-3-m & Fi-3-m & SA-1-m & PN-1-m & STR-1-m & STR-9-m & тот\% \\
\hline $\mathrm{PI} / \mathrm{PI}$ & 86 & 88 & 184 & 41 & 103 & 98 & 71.77 \\
\hline $\mathrm{Px} / \mathrm{Px}$ & 13 & 26 & 65 & 8 & 13 & 9 & 16.03 \\
\hline Ol/OI & 15 & 11 & 1 & 2 & 10 & 25 & 7.66 \\
\hline Op/Op & 8 & 6 & 2 & 3 & 0 & 0 & 2.27 \\
\hline $\mathrm{Hbl} / \mathrm{Hbl}$ & 10 & 2 & 0 & 6 & 0 & 0 & 2.15 \\
\hline $\mathrm{Bt} / \mathrm{Bt}$ & 0 & 1 & 0 & 0 & 0 & 0 & 0.12 \\
\hline
\end{tabular}

\subsubsection{Interfaces involving a pyroxene crystal}

$\mathrm{Px} / \mathrm{Pl}>>/ \mathrm{Px} /$ Horn $>>\mathrm{Px} / \mathrm{Bt}>>\mathrm{Px} / \mathrm{Idd}(=0)$.

6.1.4 Interfaces involving an amphibole crystal

$\mathrm{Hbl} / \mathrm{Op}>>\mathrm{Hbl} / \mathrm{Pl}>>\mathrm{Hbl} / \mathrm{Ol}>>\mathrm{Hbl} / \mathrm{Py}>>\mathrm{Hbl} / \mathrm{Bt}$.

6.1.5 Interfaces involving a phyllosilicate crystal

$\mathrm{Bt} / \mathrm{Op}>>\mathrm{Bt} / \mathrm{Pl}>>\mathrm{Bt} / \mathrm{Px}>>\mathrm{Bt} / \mathrm{Hbl}$.

\subsection{No-isostructural interfaces}

6.2.1 Glass-rich interfaces

$\mathrm{Pl} / \mathrm{Glgr}>>\mathrm{Px} / \mathrm{Glgr}>>\mathrm{Ol} / \mathrm{Glgr}>>\mathrm{Op} / \mathrm{Glgr}>>\mathrm{Hbl} /$

Glgr $>>$ Bt/Glgr $>>$ Idd/Glgr.

\subsubsection{Glass-poor or (microlitic) interfaces}

$\mathrm{Pl} /$ Migr $>>$ Op/Migr $>>$ Px/Migr $>>$ Ol/Migr.

\subsection{Isomineralic interfaces}

$\mathrm{Pl} / \mathrm{Pl}>>\mathrm{Px} / \mathrm{Px}>>\mathrm{Ol} / \mathrm{Ol}>>\mathrm{Op} / \mathrm{Op}>>\mathrm{Hbl} / \mathrm{Hbl}>>\mathrm{Bt} / \mathrm{Bt}$.

Moreover, the no-isostructural glassy-rich interfaces, have the highest percentage in almost all the three sand subfractions, and are more abundant than glass-poor (microlitic) interfaces. The heterogeneous glassy interfaces have revealed high proportions in the lithic grains at both beach and crater environments sand, despite their incongruence of structures and bonding types. These no-isostructural interfaces, even if experiencing high energy transport processes, proved to be both grain-size- and sedimentary environment-independent, thus they could be considered powerful as provenance- diagnostic interface types, from mafic, ultramafic and intermediate volcanic source rock lithotypes, with lathwork or porphyritic textures. The persistence of the $\mathrm{Pl} /$ Glgr, Px/Gl and Ol/Glgr, in almost all the subfractions of the islands sand, suggest that removal of glassy groundmass and breakdown along the contact with the phenocrysts proceeds at rates lower than those from tropical environments. It is likely that the comminution and mechanical damage of these interfaces - despite their vulnerability to currents and wave - is a result of inter-grains abrasion during transport, producing mainly smoothing and rounding of edges which preserve the original detrital composite signature and major portion of the entire volcanic grain area, producing once again composite lithic grains finer in size. By contrast, isomineralic interface types such as $\mathrm{Pl} / \mathrm{Pl}, \mathrm{Px} / \mathrm{Px}, \mathrm{Hbl} / \mathrm{Hbl}$, are less persistent with decreasing sand grain-size. In this case it is probable that comminution process of the isomineralic interfaces is controlled by tensile stress promoting fracturing, both along the intercrystalline interface and through intracrystals weakness planes, such as cleavage, zonation and twinning, producing monomineralic grains, similar to breakage process of glacial sand during grain saltation. As regards interfaces deforming style, associated with transport stress fields, acting on interface categories, the no-isostructural interfaces types, subjected to abrasive shear stress survive and preserve their compositional volcanic signature (Fig. 11). Abrasion produces mainly smoothing and rounding of edges which preserve the original detrital composite 


\section{INTERFACES BREAKAGE PROCESS}

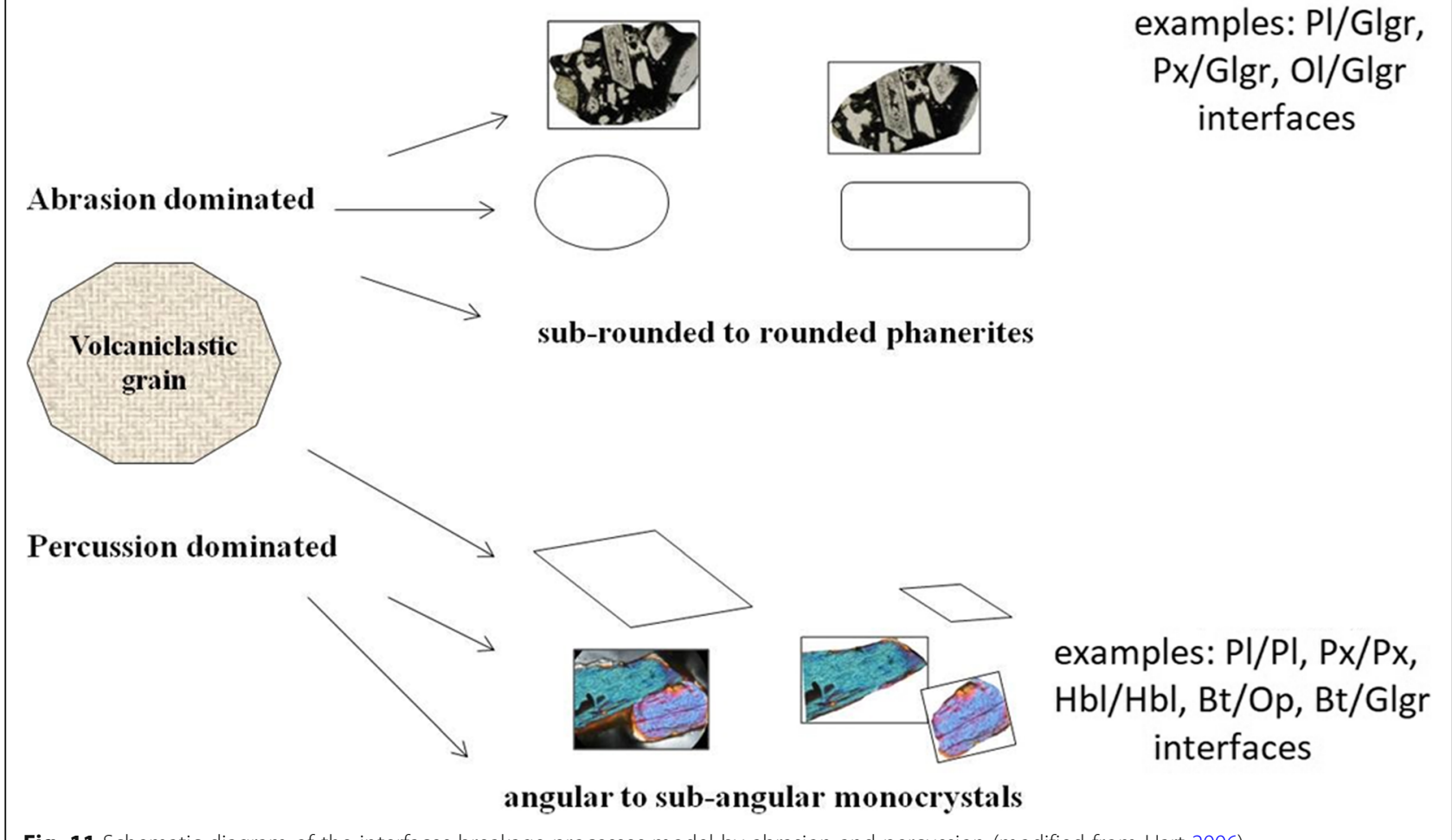

Fig. 11 Schematic diagram of the interfaces breakage processes model by abrasion and percussion (modified from Hart 2006)

signature and major portion of the entire volcanic grain area (Pl/Glgr, Px/Glgr, Ol/Glgr, Pl/Migr, etc.). By contrast, comminution process of the iso-mineralic interfaces $(\mathrm{Pl} / \mathrm{Pl}, \mathrm{Ol} / \mathrm{Ol}, \mathrm{Px} / \mathrm{Px}$, etc.) is controlled by tensile stress promoting fracturing, both along the intercrystalline interface and through intracrystals weakness planes, such as cleavage, zonation and twinning, producing monomineralic grains, similar to breakage process of glacial sand during grain saltation, in this latter case provenance signal may be overprinted by the transport processes.

In general, the interfaces types abundance and ability to survive during transport processes, can be summarized as follows: no-isostructural glass-rich>> glass-poor interfaces and no-isomineralic $>>$ iso-mineralic interfaces, from the highest to the lowest mechanical durability.

Moreover, four transport-controlled interfaces groups can may be distinguished as: ultrastable no-isostructural glass-rich interfaces, stable glass-poor interfaces, moderately stable no-isomineralic interfaces, and unstable isomineralic interfaces.

The approach of using interfacial mineralogy data to quantitatively analyze beach environment control is sound only if we are aware that, especially for volcaniclastic particles of the stratigraphic record, interfaces types abundance and diversity are controlled by pre-burial transport processes that bias compositions towards glassy-rich volcaniclastic interfaces, and with an underestimation of the isomineralic ones.

\section{Abbreviations}

Op: Opaque; Glgr: Glassy groundmass; Pl: Plagioclase; Px: Pyroxene; Ol: Olivine; Hbl: Hornblende; Idd: Iddingsite; Bt: Biotite; Rt: Rutile; Migr: Microlitic groundmass

\section{Acknowledgements}

The authors thank professors Z-Z. Feng, J. Arribas, M. Moretti and an anonymous reviewer for careful reviews and many useful suggestions which led to improvements of the manuscript.

\section{Authors' contributions}

ELP and CM contributed to the conception of the study, acquired the samples in the field, performed the research, and wrote the manuscript. Both authors contributed to read and to approve the final manuscript.

\section{Funding}

This study was funded by MIUR (Ministero dell'Istruzione, dell'Università e della Ricerca), ex 60\% fund (2019 year) to E. Le Pera.

Availability of data and materials

All data generated and analyzed during this research are included in this published article.

Competing interests

The authors declare that they have no competing interests. 
Received: 6 March 2020 Accepted: 8 July 2020

\section{Published online: 12 August 2020}

\section{References}

Andò, S., and E. Garzanti. 2013. Raman spectroscopy in heavy-mineral studies. In Sediment Provenance Studies in Hydrocarbon Exploration and Production, ed. R. A. Scott, H.R. Smyth, A.C. Morton, and N. Richardson, 395-412. Geological Society of London Special Publication 386. https://doi.org/10.1144/SP386.2.

Andò, S., E. Garzanti, M. Padoan, and M. Limonta. 2012. Corrosion of heavy minerals during weathering and diagenesis: A catalogue for optical analysis. Sedimentary Geology 280: 165-178.

Apollaro, C., F. Perri, E. Le Pera, I. Fuoco, and T. Critelli. 2019. Chemical and minero-petrographical changes on granulite rocks affected by weathering processes. Frontiers in Earth Science 13: 247-261.

Balluffi, R.W., S.M. Allen, and W.C. Carter. 2005. Structure of crystalline interfaces in kinetics of materials, 591. Wiley. 672 pp.

Barberi, F., F. Innocenti, G. Ferrara, J. Keller, and L. Villari. 1974. Evolution of Eolian arc volcanism southern Tyrrhenian Sea. Earth and Planetary Science Letters 21: 269-276.

Barros dos Santos, J.C., E. Le Pera, V.S. Souza Júnior, M.M. Corrêa, and A.C. Azevedo. 2017. Gneiss saprolite weathering and soil genesis along an east-west regolith sequence (NE Brazil). Catena 150: 279-290.

Barros dos Santos, J.C., E. Le Pera, V.S. Souza Júnior, C. Souza de Oliveira, J. Juilleret, M. Metri Corrêa, and A.C. de Azevedo. 2018. Porosity and genesis of clay in gneiss saprolites: The relevance of saprolithology to whole regolith pedology. Geoderma 319: 1-13.

Basu, A., and E. Molinaroli. 1989. Provenance characteristics of detrital opaque Fe-Ti oxides minerals. Journal of Sedimentary Petrology 59: 922-934.

Basu, A., and E. Molinaroli. 1991. Reliability and application of detrital Fe-Ti oxide minerals in provenance determination. Geological Society of London, Special Publications 57: 55-65.

Cameron, K.L., and H. Blatt. 1971. Durabilities of sand-size schist and volcanic rock fragments during fluvial transport, Elk Creek, Black Hills, South Dakota. Journal of Sedimentary Petrology 41: 565-576.

Caracciolo, L., R. Tolosana-Delgado, E. Le Pera, H. von Eynatten, J. Arribas, and S. Tarquini. 2012. Influence of granitoid textural parameters on sediment composition: Implications for sediment generation. Sedimentary Geology 280: 93-107.

Cather, S.M., and R.L. Folk. 1991. Pre-diagenetic sedimentary fractionation of andesitic detritus in a semiarid climate: An example from the Eocene Datil group, New Mexico. In Sedimentation in Volcanic Settings, ed. R.V. Fisher and G.A. Smith, vol. 45, 211-226 Society of Economic Paleontologists and Mineralogists Special Paper.

Chiarabba, C., P. De Gori, and F. Speranza. 2008. The southern Tyrrhenian subduction zone: Deep geometry, magmatism and Plio-Pleistocene evolution. Earth and Planetary Science Letters 268: 408-423.

Critelli, S., and R.V. Ingersoll. 1995. Interpretation of neovolcanic versus palaeovolcanic sand grains: An example from Miocene deep-marine sandstone of the Topanga group (southern California). Sedimentology 42: 783-804.

Critelli, S., E. Le Pera, and R.V. Ingersoll. 1997. The effects of source lithology, transport, deposition and sampling scale on the composition of southern California sand. Sedimentology 44: 653-671.

Critelli, S., K.M. Marsaglia, and C.J. Busby. 2002. Tectonic history of a Jurassic backarc-basin sequence (the gran Cañon formation, Cedros Island, Mexico), based on compositional modes of tuffaceous deposits. Geological Society of America Bulletin 114 (5): 515-527.

Critelli, S., M. Sorriso-Valvo, and G. Ventura. 1993. Relazioni tra attività vulcanica, sedimentazione epiclastica ed evoluzione geomorfologica nell'isola di Salina (Isole Eolie). Bollettino della Societa Geologica Italiana 112: 447-470

Davies, D.K., R.K. Vessell, R.C. Miles, M.G. Foley, and S.B. Bonis. 1978. Fluvial transport and downstream sediment modifications in an active volcanic region. In Fluvial Sedimentology, ed. A.D. Miall, vol. 5, 61-84 Canadian Society of Petroleum Geologists Memoir.

De Rosa, R., H. Guillou, R. Mazzuoli, and G. Ventura. 2003. New unspiked KAr ages of volcanic rocks of the central and western sector of the
Aeolian is-land: Reconstruction of the volcanic stages. Journal of Volcanology and Geothermal Research 120: 161-178.

Dickinson, W.R. 1985. Provenance relations from detrital modes of sandstones. In Provenance of Arenites, ed. G.G. Zuffa, vol. C-148, 333-362 NATO Advanced Science Institutes.

Dutta, P.K., Z. Zhou, and P.R. dos Santos. 1993. A theoretical study of mineralogical maturation of eolian sand. In Processes Controlling the Composition of Clastic Sediments, ed. M.J. Johnsson and A. Basu, vol. 284, 203-234 Geological Society of America, Special Paper.

Eggleton, R.A. 1986. The relation between crystal structure and silicate weathering rates. In Rates of chemical weathering of rocks and minerals, ed. S.M. Coleman and D.P. Dethier, 21-40. Orlando: Academic Press.

Faccenna, C., T.W. Becker, F.P. Lucente, L. Jolivet, and F. Rossetti. 2001. History of subduction and back-arc extension in the Central Mediterranean. Geophysics Journal International 145: 809-820.

Fisk, M., and N. McLoughlin. 2013. Atlas of alteration textures in volcanic glass from the ocean basins. Geosphere 9 (2): 317-341.

Francalanci, L., F. Lucchi, J. Keller, G. De Astis, and C.A. Tranne. 2013. Eruptive, volcano-tectonic and magmatic history of the Stromboli volcano (north-eastern Aeolian archipelago). In The Aeolian Islands Volcanoes, ed. F. Lucchi, A. Peccerillo, J. Keller, C.A. Tranne, and P.L. Rossi, vol. 37, 397-471 Geological Society, London, Memoirs.

Furnes, H., N. McLoughlin, K. Muehlenbachs, N. Banerjee, H. Staudigel, Y. Dilek, M. deWit, M. Van Kranendonk, and P. Schiffman. 2008. Oceanic pillow lavas and hyaloclastites as habitats for microbial life through time - a review. In Links Between Geological Processes, Microbial Activities and Evolution of Life, ed. Y. Dilek et al., 1-68. Springer Science + Business Media B.V.

Garrels, R.M., and F.T. MacKenzie. 1971. Evolution of sedimentary rocks, 397 pp. New York: Norton.

Garzanti, E. 1986. Source rock versus sedimentary control on the mineralogy of deltaic volcanic arenites (upper Triassic, northern Italy). Journal of Sedimentary Petrology 56: 267-275.

Garzanti, E., and S. Andò. 2007a. Heavy mineral concentration in modern sands: Implication for provenance interpretation. Developments in Sedimentology 58: 517-545.

Garzanti, E., and S. Andò. 2007b. Plate tectonics and heavy minerals suites of modern sands. Developments in Sedimentology 58: 741-763.

Garzanti, E., S. Canclini, F. Moretti Foggia, and N. Petrella. 2002. Unravelling magmatic and orogenic provenances in modern sands: The back-arc side of the Apennine thrust-belt (Italy). Journal of Sedimentary Research 72: 2-17.

Goldich, S.S. 1938. A study in rock weathering. Journal of Geology 46: 17-58.

Grandstaff, D.E. 1986. The dissolution rate of forsteritic olivine from Hawaiian beach sand. In Rates of chemical weathering of rocks and minerals, ed. S. M. Colman and D.P. Dethier, 41-59. New York: Academic Press.

Gvirtzman, Z., and A. Nur. 2001. Residual topography, lithospheric structure and sunken slabs in the Central Mediterranean. Earth and Planetary Science Letters 187: 117-130.

Haraldsson, H. 1984. Relations between petrography and the aggregate properties of Icelandic rocks. Bulletin of the International Association of Engineering Geologist 30: 74-76.

Hart, J.K. 2006. An investigation of subglacial processes at the microscale from Briksdalsbreen, Norway. Sedimentology 53: 125-146.

Heins, W.A. 1993. Source rock texture versus climate and topography as controls on the composition of modern, plutoniclastic sand. Geological Society of America, Special Papers 284: 135-146.

Heins, W.A. 1995. The use of mineral interfaces in sand-sized rock fragments to infer ancient climate. Geological Society of America Bulletin 107: 113-125.

Heins, W.A., and S. Kairo. 2007. Predicting sand characterwith integrated genetic analysis. In Processes Controlling the Composition of Clastic Sediments, ed. M.J. Johnsson and A. Basu, 345-379 Geological Society of America, Special Papers 284.

Hornig-Kjarsgaard, I., J. Keller, U. Koberski, E. Stadlbauer, L. Francalanci, and R. Lenhart. 1993. Geology, stratigraphy and volcanological evolution of the island of Stromboli, Aeolian arc, Italy. Acta Vulcanologica 3: 21-68.

Ingersoll, R.V. 1990. Actualistic sandstone petrofacies: Discriminating modern and ancient source rocks. Geology 18: 733-736. 
Ingersoll, R.V., T.F. Bullard, R.L. Ford, J.P. Grimm, J.D. Pickle, and S.W. Sares. 1984. The effect of grain size on detrital modes: A test of the GazziDickinson point-counting method. Journal of Sedimentary Petrology 54: 103-116.

Ingersoll, R.V., A.G. Kretchmer, and P.K. Valles. 1993. The effect of sampling scale on actualistic sandstone petrofacies. Sedimentology 40: 937-953.

ISPRA. 2011. Istituto Superiore per la Protezione e la Ricerca Ambientale. 2011. Gli indicatori del clima in Italia nel 2010. Vol. VI, 152.

Johnsson, M.J., S.D. Ellen, and M.A. McKittrick. 1993. Intensity and duration of chemical weathering: An example from soil clays of the southern Koolau Mountains, Oahu, Hawaii. In Processes Controlling the Composition of Clastic Sediments, ed. M.J. Johnsson and A. Basu, vol. 284, 147-170, Geological Society of America, Special Papers.

Keller, J. 1982. Mediterranean island arcs. In Andesites, ed. R.S. Thorpe, 307325. Chichester: Wiley.

Keller, J., I. Hornig-Kjarsgaard, U. Koberski, E. Stadlbauer, and R. Lenhart. 1993. Geological map of the island of Stromboli. In The island of Stromboli: Volcanic history and magmatic evolution, ed. P. Manetti and J. Keller Acta Vulcanologica, 3, Appendix.

King, R.H. 1986. Weathering of Holocene airfall tephras in the southern Canadian Rockies. In Rates of chemical weathering of rocks and minerals, ed. S.M. Colman and D.P. Dethier, 239-262. New York: Academic Press.

Le Pera, E., and S. Critelli. 1997. Sourceland controls on the composition of beach and fluvial sand of the Tyrrhenian coast of Calabria, Italy: Implications for actualistic petrofacies. Sedimentary Geology 110: 81-97.

Le Pera, E., and C. Morrone. 2018. Heavy minerals distribution and provenance in modern beach sands of Campania, Italy. Rendiconti Online della Società Geologica Italiana 45: 136-140. https://doi.org/10.3301/ROL.2018.41.

Le Pera, E., and M. Sorriso-Valvo. 2000. Weathering and morphogenesis in a Mediterranean climate, Calabria, Italy. Geomorphology 34: 251-270.

Locke, W.W. 1986. Rates of hornblende etching in soils on glacial deposits, Baffin Island, Canada. In Rates of chemical weathering of rocks and minerals, ed. S.M. Colman and D.P. Dethier, 129-145. New York: Academic Press.

Lowe, D.J. 1986. Controls on the rates of weathering and clay minerals genesis in airfall tephras: A review and New Zealand case study. In Rates of chemical weathering of rocks and minerals, ed. S.M. Colman and D.P. Dethier, 265-330. New York: Academic Press.

Lucchi, F. 2009. Late-Quaternary terraced marine deposits as tools for widescale correlation of unconformity-bounded units in the volcanic Aeolian archipelago (southern Italy). Sedimentary Geology 216: 158-178.

Lucchi, F. 2013. Stratigraphic methodology for the geological mapping of volcanic areas: Insights from the Aeolian archipelago (southern Italy). In The Aeolian Islands Volcanoes, ed. F. Lucchi, A. Peccerillo, J. Keller, C.A. Tranne, and P.L. Rossi, vol. 37, 37-53 Geological Society, London, Memoirs.

Lucchi, F., R. Gertisser, J. Keller, F. Forni, G. De Astis, and C.A. Tranne. 2013c. Eruptive history and magmatic evolution of the island of Salina (central Aeolian archipelago). In The Aeolian Islands Volcanoes, ed. F. Lucchi, A. Peccerillo, J. Keller, C.A. Tranne, and P.L. Rossi, vol. 37, 155-211 Geological Society, London, Memoirs.

Lucchi, F., A. Peccerillo, C.A. Tranne, P.L. Rossi, M.L. Frezzotti, and C. Donati. 2013a. Volcanism, calderas and magmas of Alicudi composite volcano (western Aeolian archipelago). In The Aeolian Islands Volcanoes, ed. F. Lucchi, A. Peccerillo, J. Keller, C.A. Tranne, and P.L. Rossi, vol. 37, 83-111 Geological Society, London, Memoirs.

Lucchi, F., A.P. Santo, C.A. Tranne, A. Peccerillo, and J. Keller. 2013b. Volcanism, magmatism, volcano-tectonics and sea-level fluctuations in the geological history of Filicudi (western Aeolian archipelago). In The Aeolian Islands Volcanoes, ed. F. Lucchi, A. Peccerillo, J. Keller, C.A. Tranne, and P.L. Rossi, vol. 37, 113-154 Geological Society, London, Memoirs.

Lucchi, F., C.A. Tranne, A. Peccerillo, J. Keller, and P.L. Rossi. 2013d. Geological history of the Panarea volcanic group (eastern Aeolian archipelago). In The Aeolian Islands Volcanoes, ed. F. Lucchi, A. Peccerillo, J. Keller, C.A. Tranne, and P.L. Rossi, vol. 37, 349-394 Geological Society, London, Memoirs.

Mack, G.H. 1978. The survivability of labile light mineral grains in fluvial, eolian, and littoral environments: The Permian cutler-cedar Mesa formations, Moab, Utah. Sedimentology 25 (5): 587-604.
Mack, G.H., and T. Jerzykiewicz. 1989. Detrital modes of sand and sandstone derived from andesitic rocks as paleoclimatic indicator. Sedimentary Geology 65: 35-44.

Marsaglia, K.M. 1992. Petrography and provenance of volcaniclastic sands recovered from the Izu-Bonin arc, leg 126. Proceeding of the Ocean Drilling Program, Scientific Results 126: 139-154.

Marsaglia, K.M. 1993. Basaltic island sand provenance. In Processes Controlling the Composition of Clastic Sediments, ed. M.J. Johnsson and A. Basu, vol. 284, 41-65 Geological Society of America, Special Papers.

Marsaglia, K.M., and K. Tazaki. 1992. Diagenetic trend in ODP leg 126 sandstones. In Proceedings of the Ocean Drilling Program, Scientific Results 126, ed. B. Taylor, K. Fujioka, T.R. Janecec, and C. Langmuir, 125138. College Station: Ocean Drilling Program.

McCarroll, D. 1990. Differential weathering of feldspar and pyroxene in an arctic alpine environment. Earth Surface Processes and Landforms 15 (7): 641-651.

Molinaroli, E., and A. Basu. 1993. Toward quantitative provenance analysis: A brief review and case study. In Processes Controlling the Composition of Clastic Sediments, ed. M.J. Johnsson and A. Basu, vol. 284, 323-333 Geological Society of America, Special Papers.

Morrone, C., R. De Rosa, E. Le Pera, and K.M. Marsaglia. 2017. Provenance of volcaniclastic beach sand in a magmatic-arc setting: An example from Lipari island (Aeolian archipelago, Tyrrhenian Sea). Geological Magazine 154 (4): 804-828.

Morrone, C., E. Le Pera, R. De Rosa, and K.M. Marsaglia. 2018. Beach sands of Lipari island, Aeolian archipelago: Roundness study. Rendiconti Online della Società Geologica Italiana 45: 141-146. https://doi.org/10.3301/ROL. 2018.42.

Morrone, C., E. Le Pera, K.M. Marsaglia, and R. De Rosa. 2020. Compositional and textural study of modern beach sands in the active volcanic area of the Campania region (southern Italy). Sedimentary Geology 396. https:// doi.org/10.1016/j.sedgeo.2019.105567.

Palomares, M., and J. Arribas. 1993. Modern stream sands from compound crystalline sources: Composition and sand generation index. In Processes Controlling the Composition of Clastic Sediments, ed. M.J. Johnsson and A. Basu, vol. 284, 313-322 Geological Society of America, Special Papers.

Patacca, E., and P. Scandone. 1989. Post-Tortonian mountain building in the Apennines: the role of the passive sinking of a relic lithospheric slab. In The Lithosphere in Italy: Advances in Earth Science Research, ed. A.M. Boriani, M. Bonafede, G.B. Piccardo, and G.B. Vai, 157-176. Rome: Accademia Nazionale dei Lincei.

Peccerillo, A., G. De Astis, D. Faraone, F. Forni, and M.L. Frezzotti. 2013. Compositional variations of magmas in the Aeolian arc: implications for petrogenesis and geodynamics. In The Aeolian Islands Volcanoes, ed. F. Lucchi, A. Peccerillo, J. Keller, C.A. Tranne, and P.L. Rossi, vol. 37, 491-510 Geological Society, London, Memoirs.

Pettijohn, F.P., P.E. Potter, and R. Siever. 1987. Sand and Sandstones. 2nd ed, 583. New York: Springer-Verlag.

Pittman, E.D. 1969. Destruction of plagioclase twins by stream transport. Journal of Sedimentary Petrology 39: 1432-1437.

Riber, L., H. Dypvik, and R. Sørlie. 2015. Altered basement rocks on the Utsira high and its surroundings, Norwegian North Sea. Norvegian Journal of Geology 95 (1): 57-89.

Riber, L., H. Dypvik, R. Sørlie, and R.E. Ferrell. 2016. Clay minerals in deeply buried paleoregolith profiles, Norwegian North Sea. Clays and Clay Minerals 64 (5): 488-508.

Riber, L., H. Dypvik, R. Sørlie, S.A.A.M. Naqvi, K. Stangvik, N. Oberhardt, and P. A. Schroeder. 2017. Comparison of deeply buried paleoregolith profiles, Norwegian North Sea, with outcrops from southern Sweden and Georgia, USA_Implications for petroleum exploration. Palaeogeography, Palaeoclimatology, Palaeoecology 471: 82-95.

Sætre, C., H. Hellevang, C. Dennehy, H. Dypvik, and S. Clark. 2018. A diagenetic study of intrabasaltic siliciclastics sandstones from the Rosebank field. Marine and Petroleum Geology 98: 335-355.

Sætre, C., H. Hellevang, L. Riud, H. Dypvik, C. Pilorget, F. Poulet, and S.C. Werner. 2019. Experimental hydrothermal alteration of basaltic glass with relevance to Mars. Meteoritics and Planetary Science 54: 357-378. 
Scarciglia, F., N. Saporito, M.F. La Russa, E. Le Pera, M. Macchione, D. Puntillo, G.M. Crisci, and A. Pezzino. 2012. Role of lichens in weathering of granodiorite in the Sila uplands (Calabria, southern Italy). Sedimentary Geology 280: 119-134.

Slatt, R.M., and N. Eyles. 1981. Petrology of glacial sand: Implications for the origin and mechanical durability of lithic fragments. Sedimentology 28 (2): 171-183.

Smith, G.A., and J.E. Lotosky. 1995. What factors control the composition of andesitic sand? Journal of Sedimentary Research A65 (1): 91-98.

Tranne, C.A., F. Lucchi, N. Calanchi, G. Lanzafame, and P.L. Rossi. 2002a. Geological map of the island of Lipari (Aeolian Islands) (scale 1:12.500). Firenze: University of Bologna and INGV, LAC.

Tranne, C.A., F. Lucchi, N. Calanchi, G. Lanzafame, and P.L. Rossi. 2002b. Geological map of the island of Filicudi (Aeolian Islands) (scale 1:10.000). Firenze: University of Bologna and INGV, LAC.

Velbel, M.A. 1989. Weathering of hornblende to ferruginous products by a dissolution reprecipitation mechanism: Petrography and stoichiometry. Clays and Clay Minerals 37 (6): 515-524.

Velbel, M.A. 1999. Bond strength and the relative weathering rates of simple orthosilicates. American Journal of Science 299: 679-696.

Velbel, M.A. 2009. Dissolution of olivine during natural weathering. Geochimica et Cosmochimica Acta 73: 6098-6113.

Velbel, M.A. 2014. Etch-pit size, dissolution rate, and time in the experimental dissolution of olivine: Implications for estimating olivine lifetime at the surface of Mars. American Mineralogist 99: 2227-2233.

Velbel, M.A., and W.W. Barker. 2008. Pyroxene weathering to smectite: Conventional and cryo-field emission scanning electron microscopy, Koua Bocca ultramafic complex, Ivory Coast. Clays and Clay Minerals 56 (1): 112-127.

Ventura, G. 2013. Kinematics of the Aeolian volcanism (southern Tyrrhenian Sea) from geophysical and geological data. In The Aeolian Islands Volcanoes, ed. F. Lucchi, A. Peccerillo, J. Keller, C.A. Tranne, and P.L. Rossi, vol. 37, 3-11 Geological Society, London, Memoirs.

Ventura, G., G. Vilardo, G. Milano, and N.A. Pino. 1999. Relationships among crustal structure, volcanism and strike-slip tectonics in the Lipari-Vulcano volcanic complex Aeolian Islands, southern Tyrrhenian Sea, Italy. Physics of the Earth and Planetary Interiors 116: 31-52.

Villari, L. 1980. The island of Alicudi. Rendiconti della Societa Italiana di Mineralogia e Petrologia 36: 441-466.

Weltje, G.J. 2012. Quantitative models of sediment generation and provenance: State of the art and future developments. Sedimentary Geology 280: 4-20.

Weltje, G.J., B. Paredis, L. Caracciolo, and W.A. Heins. 2018. Quantitative analysis of crystal-interface frequencies in granitoids: Implications for modelling of parent-rock texture and its influence on the properties of plutoniclastic sands. Sedimentary Geology 375: 72-85.

White, A.F., L.V. Benson, and A. Yee. 1986. Chemical weathering of the May 18, 1980, Mount St. Helens ash fall and the effect on the Iron Creek watershed, Washington. In Rates of chemical weathering of rocks and minerals, ed. S.M. Colman and D.P. Dethier, 351-374. New York: Academic Press.

Williams, H. 1950. Volcanoes of the Paricutin region, Mexico. United States Geological Survey Bulletin 965-B: 165-279.

\section{Publisher's Note}

Springer Nature remains neutral with regard to jurisdictional claims in published maps and institutional affiliations.

\section{Submit your manuscript to a SpringerOpen ${ }^{\circ}$ journal and benefit from:}

- Convenient online submission

- Rigorous peer review

- Open access: articles freely available online

- High visibility within the field

- Retaining the copyright to your article

Submit your next manuscript at $\boldsymbol{\nabla}$ springeropen.com 